This is an Accepted Manuscript of an article published by Taylor \& Francis in Journal of Personality Assessment on 1 October 2021, available online:

\title{
https://www.tandfonline.com/doi/full/10.1080/00223891.2021.1981346
}

A Critical Evaluation of the Reflective Functioning Questionnaire (RFQ)

Sascha Müller ${ }^{*}$, Leon P. Wendt ${ }^{1 *}$, Carsten Spitzer², Oliver Masuhr ${ }^{3}$, Sarah N. Back ${ }^{4}$, \& Johannes Zimmermann ${ }^{1}$

\footnotetext{
${ }^{1}$ University of Kassel, Department of Psychology, Kassel, Germany

${ }^{2}$ Rostock University Medical Center, Rostock, Germany

${ }^{3}$ Asklepios Fachklinikum Tiefenbrunn, Rosdorf, Germany

${ }^{4}$ Ludwig-Maximilians-Universität München, Munich, Germany

*shared first authorship
}

Authors' note: Ethics committee approval was obtained for data collection (Ethics Committee at the University Medicine Rostock, \#A2020-0075; Ethics Committee at the Faculty of Behavioural and Cultural Studies, University of Heidelberg, \#Tau 2019 1/1). The first authors contributed equally to this research. The data used in Study 1 are available from the corresponding authors upon reasonable request. Data and $\mathrm{R}$ code for reproducing the analyses of Study 2 are permanently and openly accessible at https://osf.io/stbz5. Please address correspondence to Sascha Müller and Leon Wendt, Department of Psychology, University of Kassel, Holländische Str. 36-38, 34127, Kassel, Germany; E-Mail: sascha.mueller@unikassel.de, 1.wendt@uni-kassel.de. 


\begin{abstract}
The Reflective Functioning Questionnaire (RFQ) is an 8-item self-report measure of reflective functioning that is presumed to capture individual differences in hypo- and hypermentalizing. Despite its broad acceptance by the field, we argue that the validity of the measure is not well-established. The current research elaborates on problems of the RFQ related to its item content, scoring procedure, dimensionality, and associations with psychopathology. We tested these considerations across three large clinical and non-clinical samples from Germany and the US (total $N=2289$ ). In a first study, we found that the RFQ may assess a single latent dimension related to hypomentalizing but is rather unlikely to capture maladaptive forms of hypermentalizing. Moreover, the RFQ exhibited very strong associations with measures of personality pathology, while associations with measures of symptom distress were less strong. In a second preregistered study focused on convergent and discriminant validity, however, a commonality analysis indicated that associations with indicators of personality pathology are inflated because some of the RFQ items tap into emotional lability and impulsivity rather than mentalizing. Our findings demonstrate limitations of the RFQ. We discuss key challenges in assessing mentalizing via self-report.
\end{abstract} Keywords: reflective functioning; mentalizing; validity; factor analysis; U-shaped associations; commonality analysis 


\section{A Critical Evaluation of the Reflective Functioning Questionnaire (RFQ)}

Fonagy and colleagues (2016) introduced the Reflective Functioning Questionnaire (RFQ), a brief screening measure that is intended to assess an individual's capacity to adequately interpret mental states of both the self and others (i.e., reflective functioning or mentalizing) via self-report. The measure as well as its translated versions have been positively evaluated in several validation studies (Badoud et al., 2015; Fonagy et al., 2016; Morandotti et al., 2018), leading to the consensual conclusion that the RFQ is able to capture deficits in reflective functioning. According to mentalizing theory (e.g., Bateman \& Fonagy, 2019), the theoretical spectrum of reflective functioning includes hypo- and hypermentalizing (i.e., too little or too much certainty about one's interpretation of mental states) as well as genuine mentalizing (the optimal trait level, i.e., acknowledging the opaqueness of mental states). Deviations from the optimal trait level in both directions are presumed to be a sign, a symptom, and a transdiagnostic risk factor of psychopathology (e.g., Luyten et al., 2020) and have been targeted by a specialized psychotherapeutic approach, that is, mentalization-based treatment (e.g., Bateman \& Fonagy, 2016).

In the RFQ, respondents are asked to endorse or reject eight statements that are supposed to relate to mentalizing processes (e.g., Item 6; "Sometimes I do things without really knowing why") on a 7-point scale ranging from do not agree at all $(=1)$ to agree completely $(=7)$ (see Panel A of Figure 1). The creators implemented a procedure that involves scoring half of the items twice (i.e., double-scoring) and splitting the underlying information into two scales (see Panel B of Figure 1): certainty about mental states (RFQ_C; i.e., 3, 2, 1, 0, 0, 0, 0) and uncertainty about mental states (RFQ_U; i.e., 0, 0, 0, 0, 1, 2, 3). According to this logic, a strong rejection of Item 6 on the original scale $(=1)$ is scored in such a manner that it is indicative of high certainty $(=3)$ on the RFQ_C scale and at the same time indicative of low uncertainty $(=0)$ on the RFQ_U scale, while a strong agreement is 
scored as indicative of low certainty $(=0)$ on the RFQ_C scale and at the same time as indicative of high uncertainty $(=3)$ on the RFQ_U scale. High levels of certainty about mental states are assumed to reflect hypermentalizing and high levels of uncertainty are assumed to reflect hypomentalizing (e.g., Badoud et al., 2015; Fonagy et al., 2016; Morandotti et al., 2018). The latent structure of the RFQ has been investigated repeatedly using double-scored items and reportedly consists of two negatively correlated factors (with correlations typically ranging between -.60 and -.80) reflecting the scales of RFQ_C and RFQ_U (Badoud et al., 2015; Fonagy et al., 2016).

However, in a recent attempt to validate the factor structure of the German version of the RFQ in a large sample $(N=2477)$ representative of the German general population, Spitzer et al. (2021) criticized the use of double-scored items in factor analysis (e.g., Badoud et al., 2015; Fonagy et al., 2016; Morandotti et al., 2018), noting that the assumption of uncorrelated item residuals seems unrealistic when two items are derived from the same original responses. Instead, Spitzer and colleagues provided initial evidence that a unidimensional model sufficiently explained the observed covariation of the original responses to RFQ items and suggested that this representation could give rise to two maladaptive poles of hypo- and hypermentalizing deviating from an adaptive middle region. Following this notion, they proceeded with testing U-shaped relationships between a unidimensional RFQ score (dropping two items to improve internal consistency) and depression, anxiety, and somatic symptoms but found no evidence for such associations. The findings of Spitzer and colleagues in conjunction with their briefly noted considerations raise some initial doubts and call for a critical re-examination of the RFQ as a psychometric test. A critical re-examination and discussion of the RFQ appears to be of particular importance given that researchers are increasingly adopting the measure for primary investigations of the mentalizing construct (e.g., Badoud et al., 2018; de Meulemeester et al., 2017, 2018; Huang 
et al., 2020; Li et al., 2020). In the following, we will identify and explore potential issues with the RFQ in detail.

\section{Item Content}

An initial concern is that the coverage of the reflective functioning construct in the RFQ does not seem to converge well with the definition given by the creators (Fonagy et al., 2016). According to them, reflective functioning pertains to "the capacity to interpret both the self and others in terms of internal mental states, such as feelings, wishes, goals, desires, and attitudes" (Fonagy et al., 2016, p. 1). However, all RFQ items but one refer to understanding oneself (and not others) and most items refer to understanding one's own behavior (and not feelings, desires, wishes, goals, or attitudes). The construct of reflective functioning as defined by the authors might thus not be optimally covered by the items. Although it should be acknowledged that the RFQ was introduced as a brief screening measure and the creators themselves generally called for a multidimensional assessment of mentalizing (e.g., Fonagy et al., 2016; Luyten et al., 2020), a potential lack of coverage cannot be compensated by relating the RFQ to a validated and more comprehensive long form. This is because, even though long forms (i.e., RFQ-54, RFQ-46) are accessible from the creators' website and are also used in some studies (e.g., Euler et al., 2021), there are no publications that investigate their psychometric properties or their relationship to the brief RFQ.

In addition, it seems that the item content of the RFQ may overrepresent another maladaptive characteristic, namely, a tendency towards impulsive behavior when experiencing negative emotions (e.g., Item 3: "When I get angry, I say things without really knowing why I am saying them"; Item 4: "When I get angry, I say things that I later regret"; Item 5: "If I feel insecure I can behave in ways that put others' backs up"). That is, it could be that individuals who endorse these items are not necessarily lacking in reflective capacity, but merely show a high level of negative urgency (Cyders \& Smith, 2008; Settles et al., 2012). 
Negative urgency denotes the disposition to act rashly and ill-advisedly under negative emotions and can thus be seen as reflecting a blend of emotional lability and impulsivity. While this behavioral signature could be considered a consequence of impairments in mentalizing abilities, it may well be due to other underlying characteristics and deficiencies (e.g., lack of adaptive emotion regulation strategies; King et al., 2018). Therefore, these items do not seem specific enough to the core definition of reflective functioning. By contrast, other items of the RFQ such as "People's thoughts are a mystery to me" (Item 1) or "I always know what I feel" (Item 7) seem to address more directly the certainty or uncertainty involved in forming inferences about mental states.

\section{Scoring Procedure}

The second concern pertains to the aforementioned double-scoring of four of the eight RFQ items (i.e., Items 2, 4, 5, 6) to derive RFQ_U and RFQ_C scale scores and their use in factor analysis, as it causes psychometric problems. Given that respondents only provide one rating for each of these four items on the 7-point scale, the resulting eight rescaled scores on RFQ_C and RFQ_U are mutually determined. For example, when a respondent rates Item 6 with strong agreement, this necessarily leads to a rescaled score of 3 on RFQ_C and a rescaled score of 0 on RFQ_U (see Panels A and B of Figure 1). Thus, the two rescaled scores are not independent of each other and overlap with regard to their information. In fact, nine of the 16 theoretical combinations of the two scores are mathematically impossible (see Panel C of Figure 1). In our Sample 1 (see below), this results in an artificially negative correlation between the two rescaled scores of Item 6 with $r=-.55$. The exact value of the correlation depends on the univariate distribution of the raw scores and varies slightly between items and samples. However, when using polychoric correlations that take into account the ordinal scaling of the scores, the estimate will always be exactly $r=-1$. This shows that the two rescaled scores are in fact completely redundant. This issue is particularly 
problematic because the assumed factor structure of the RFQ is based on confirmatory factor analysis (CFA) using double-scored items as indicators (Badoud et al., 2015; Fonagy et al., 2016). Applying ordinal CFA should produce warning messages because the polychoric correlations between several indicators will approach $r=-1$. However, if instead one treats the indicators as continuous and applies robust maximum likelihood estimation (as was done in previous validation studies), one also cannot undo the inherent dependencies of the indicators. In this case, the suggested two-dimensional factor model will artificially induce a negative correlation between the two factors because the residual correlations of doublescored item pairs are restricted to zero. Therefore, the finding of two negatively correlated dimensions of the RFQ (i.e., RFQ_U and RFQ_C) using double-scored items should rather not be interpreted as evidence in favor of the instrument's structural validity as was done in previous validation studies (Badoud et al., 2015; Fonagy et al., 2016). We argue that the factor structure of the RFQ is still open to debate.

The problematic scoring procedure associated with the two scales manifests itself in conceptual inconsistencies with regard to the RFQ_C scale in particular. Although RFQ_C is supposed to represent certainty about mental states, all items are geared towards a state of uncertainty with respect to their semantic content (e.g., Item 1: "People's thoughts are a mystery to me") and are, ultimately, reversely scored. The certainty scale is thus based entirely on the denial of uncertainty. In fact, the RFQ contains only one item directly referring to a state of certainty (Item 7: "I always know what I feel") and this item is scored exclusively for the uncertainty scale (RFQ_U).

\section{Associations with Psychopathology}

A third issue is that findings on associations between RFQ scales and psychopathology constructs are somewhat conflicting. As theory posits that mentalizing impairments such as hypo- and hypermentalizing are a vulnerability factor for severe 
psychopathology (e.g., Luyten et al., 2020), positive associations between mentalizing impairments and various indicators of psychopathology are expected. However, the correlational patterns of the RFQ_C and RFQ_U scales that emerge from the literature (e.g., Badoud et al., 2015; Fonagy et al., 2016; Huang et al., 2020; Li et al., 2020) rather appear to be in contrast to the interpretation that RFQ_C assesses hypermentalizing, as has been noted previously (de Meulemeester et al., 2018; Euler et al., 2021). Overall, the RFQ_C scale (certainty about mental states) was often positively associated with mental health, suggesting that it captures an adaptive characteristic. By contrast, the RFQ_U scale (uncertainty about mental states) appears to be quite strongly related to various indices of psychopathology. Taken together, the two scales of the RFQ tend to exhibit similar correlational patterns to external criteria but with opposite signs, respectively. These opposing correlational patterns of RFQ_C and RFQ_U in a shared nomological net seem more compatible with the notion that the RFQ reflects a unidimensional continuum ranging from genuine to impaired mentalizing.

Another aspect in this regard is that, from a theoretical point of view, the association between mentalizing and psychopathology should depend on which form of psychopathology is being considered. Specifically, impairments in mentalizing are thought to be a core feature of personality disorders (e.g., Bateman \& Fonagy, 2019; Fonagy et al., 2017; Luyten et al., 2020). For example, in the Alternative Model for Personality Disorders (AMPD) in the fifth edition of the Diagnostic and Statistical Manual of Mental Disorders (DSM-5; APA, 2013), the first general criterion of personality disorders refers to difficulties in understanding and regulating the self and interpersonal relationships, with mentalizing deficits explicitly considered as one important element (Bender et al., 2011). Thus, the strongest correlations in the field of psychopathology should occur where the RFQ is correlated with measures that capture the severity of personality pathology. Previous studies documented strong 
associations between the RFQ scales and measures of personality pathology (e.g., Badoud et al., 2015; de Meulemeester et al., 2017; Fonagy et al., 2016) as well as various symptom measures (e.g., de Meulemeester et al., 2018; Fonagy et al., 2016; Huang et al., 2020; Li et al., 2020). However, the magnitudes of these associations should be compared systematically by inferential testing. Notably, differential associations should be carefully interpreted in terms of validity, not least because the RFQ also contains item content that may reflect constructs such as negative urgency rather than mentalizing in particular. For example, impulsive responsivity to emotions may be a general underlying disposition for psychological distress that would be expected to exhibit similar patterns of associations as were reported for the scales of the RFQ (e.g., Carver et al., 2017; Settles et al., 2012). We therefore argue that more research is needed to evaluate whether associations of the RFQ with specific, theoretically selected forms of psychopathology are higher than associations with nonspecific symptom distress. In addition, it should be investigated whether associations between the RFQ and indicators of personality dysfunction are artificially inflated due to item content that is shared with other maladaptive characteristics.

\section{Study 1}

We have stated potential concerns with regard to the RFQ related to its item content, scoring procedure, dimensionality, and associations with psychopathology. Based on our interpretation of previous findings, we make three observations: First, we propose that the measure may emerge as unidimensional when modeled more adequately, that is, when not using double-scored items in CFA given the methodological problems associated with this approach. Although initial evidence for the unidimensionality of the RFQ has been provided by Spitzer et al. (2021), their study only involved a single non-clinical sample. In the first study, we used data from a clinical and a non-clinical sample to compare a series of factor 
models for the RFQ including unidimensional and two-dimensional representations in order to test the generalizability and robustness of a unidimensional solution.

Second, if the RFQ turns out to be unidimensional, it could still capture a unipolar or bipolar construct. As a unipolar construct, it could range from genuine to impaired mentalizing, whereas as a bipolar construct, it could capture hypo- and hypermentalizing as two maladaptive ends of one continuum. Notably, in our view, a unidimensional scoring of the RFQ lends itself to investigating the unique characteristics of the two poles using nonlinear statistical models. Specifically, in the case where the RFQ captures a continuum that runs from extreme uncertainty about mental states ("too little mentalizing") to an optimal level ("genuine mentalizing") to excessive certainty about mental states ("too much mentalizing"), one would predict U-shaped associations with maladaptive characteristics (e.g., depression) and, vice versa, inverse U-shaped associations with adaptive characteristics (e.g., well-being). ${ }^{1}$ When first testing this hypothesis by specifying quadratic terms in regression models to predict psychopathology outcomes, Spitzer et al. (2021) found no support for such U-shaped associations. However, the authors considered only rather weak criteria in terms of short screening measures of depression, anxiety, and somatic symptoms. In this study, we explored (inverse) U-shaped relationships between the RFQ and measures indicative of psychopathology and personality pathology to provide more comprehensive tests of the hypothesis that the RFQ captures two maladaptive variants of mentalizing on a single continuum (i.e., hypo- and hypermentalizing).

Third, given that the RFQ contains several non-specific items, we examined associations with specific forms of psychopathology that are theoretically more closely

\footnotetext{
${ }^{1}$ Thus, both low and high levels of the unidimensional RFQ are supposed to be maladaptive, marking the high ends of the U-shape when inspecting a maladaptive criterion. By contrast, middle levels are not supposed to be maladaptive, marking the turning point of the U-shape when inspecting a maladaptive criterion. Similarly, the association between the RFQ and adaptive criteria should be inverse U-shaped, indicating that middle levels are adaptive whereas both low and high levels are not.
} 
related to the core construct of mentalizing. We therefore selected several measures of personality pathology that are linked to Criterion A (impairments in personality functioning) and Criterion B (maladaptive personality traits) of the AMPD. We compared the correlations between the RFQ and measures of personality pathology with correlations between the RFQ and measures of general symptom distress, and further scrutinized the latent associations between the RFQ and personality pathology using bifactor exploratory structural equation modeling (bifactor ESEM).

\section{Method}

Sample 1. The first sample was collected at a psychosomatic clinic, the Asklepios Fachklinikum Tiefenbrunn. Participants (64\% female) were 861 inpatients ranging in age from 18 to $68(M=34.0, S D=13.3)$. The data presented here were collected from patients who were admitted for inpatient treatment within three days of admission and shortly before discharge as part of routine diagnostics. The RFQ was administered to all included participants at admission without missings, whereas only 364 participants completed the measure at discharge. The reason for these missing observations is not dropout; the RFQ was only administered at discharge at a later stage of data collection and thus only pertained to 364 patients. For more detailed information about the sample characteristics, see Note S1 in the supplement.

Sample 2. The second sample was collected online within a study on dimensional measures of personality at Heidelberg University Hospital (Back et al., 2020). Participants were recruited via flyers and calls for participation on the university website and in several online forums. The sample comprises 566 young adults ( $74 \%$ female) who completed the study and whose ages ranged from 18 to $30(M=24.2, S D=3.13)$. There were no missing data. For more detailed information about the sample characteristics, see Note S2 in the supplement. 
Measures. The RFQ was administered in both samples. The Brief Symptom Inventory (BSI), Inventory of Interpersonal Problems (IIP-32), WHO-5 Well-Being Index (WHO-5), Patient Health Questionnaire (PHQ), Inventory of Personality Organization (IPO16), and Operationalized Structural Diagnostic Questionnaire - Short Form (OPD-SQS) were administered in Sample 1. Some of the criterion measures were administered at both admission and discharge in Sample 1; however, we used only the data at admission (i.e., before treatment) for exploring their associations with the RFQ. The Personality Inventory for DSM-5 - Brief Form (PID-5-BF) was administered in Sample 2.

Reflective Functioning Questionnaire (RFQ). The RFQ (Fonagy et al., 2016) comprises eight items forming the two scales of certainty about mental states (RFQ_C) and uncertainty about mental states (RFQ_U). According to the scoring procedure described above, the 7-point Likert scale ("do not agree at all" = 1 to "agree completely" $=7$ ) is recoded as 3, 2, 1, 0, 0, 0, 0 for the scale RFQ_C; for the scale RFQ_U, items are recoded as $0,0,0,0,1,2,3$ (except for the inverse Item 7 that is recoded as shown for RFQ_C). Results for the original scoring procedure are supplemented. For the main results, we refrained from applying the scoring procedure due to the aforementioned problems. Items were thus kept in the original coding (i.e., 1, 2, 3, 4, 5, 6, 7) and only Item 7 was reversed such that it corresponded to the content polarity of the other items. High values indicated uncertainty about mental states and low values indicated certainty. In the following, we will refer to the mean score of the 8-item RFQ as the RFQ-8. In a previous investigation using a representative sample from the German population, Spitzer et al. (2021) presented results in favor of using a reduced 6-item version of the RFQ (omitting Items 7 and 4), that will subsequently be referred to as RFQ-6.

Brief Symptom Inventory (BSI). The BSI (Franke, 2000) was used to assess distress associated with symptoms of mental illness during the last week (e.g., "loss of appetite") on a 
5-point scale ranging from "not at all" (0) to "extremely" (4). Internal consistency of the BSI total score was estimated at $\alpha=.95$ in Sample 1 .

Inventory of Interpersonal Problems (IIP-32). Interpersonal problems were measured with the 32-item version of the IIP (Horowitz et al., 2000). The measure assesses distress associated with interpersonal behaviors (e.g., "I open up to other people too much") that are performed excessively or inhibited strongly on a 5-point scale ranging from "not at all" (0) to "extremely" (4). Internal consistency of the IIP-32 total score was estimated at $\alpha=$ .87 in Sample 1.

WHO-5 Well-Being Index (WHO-5). The WHO-5 (World Health Organization, 1998 ) is a self-report measure of well-being. It consists of five items (e.g., "Over the last two weeks I have felt cheerful and in good spirits") that are rated on a 6-point Likert scale ranging from "at no time" (0) to "all the time" (5). High scores indicate a high subjective well-being. Internal consistency of the WHO-5 total score was estimated at $\alpha=.85$ in Sample 1 .

Patient Health Questionnaire (PHQ). The PHQ-15 (Kroenke et al., 2002) is a 15item module for assessing the severity of impairment associated with somatic symptoms (e.g., "back pain") that have been experienced during the last four weeks. Items are rated on a 3-point scale ranging from "not bothered at all" (0) to "bothered a lot" (2). The PHQ-9 (Kroenke et al., 2001) is a 9-item module for assessing the impairment associated with the nine DSM-IV criteria of depression (e.g., "Little interest or pleasure in doing things", "Feeling tired or having little energy") that may have been experienced during the last two weeks. Items are rated on a 4-point scale ranging from "not at all" (0) to "nearly every day" (3). Internal consistency of the PHQ-15/PHQ-9 total scores were estimated at $\alpha=.81 / .84$ in Sample 1.

Inventory of Personality Organization (IPO-16). The IPO-16 assesses general personality dysfunction (Zimmermann et al., 2013) in the domains of identity diffusion (e.g., 
"I feel that my tastes and opinions are not really my own, but have been borrowed from other people"), primitive defenses (e.g., "People tell me I behave in contradictory ways"), and reality testing (e.g., "I can't tell whether certain physical sensations I'm having are real, or whether I'm imagining them"). The 16 items are answered on a 5-point scale from "never applies" (1) to "always applies" (5). Internal consistency of the IPO-16 total score was estimated at $\alpha=.86$ in Sample 1 .

\section{Operationalized Psychodynamic Diagnosis - Structure Questionnaire Short Form}

(OPD-SQS). The OPD-SQS is a 12-item measure of personality dysfunction (Ehrenthal et al., 2015). Statements are endorsed or rejected on a 5-point scale ranging from "completely untrue" (0) to "entirely true" (4). The items give rise to the scales of self-perception (e.g., "I sometimes feel like a stranger to myself”), contact (e.g., "I sometimes misjudge how my behavior affects others"), and relationship (e.g., "It can be dangerous to let others get too close to you."). Internal consistency of the OPD-SQS total score was estimated at $\alpha=.86$ in Sample 1.

Personality Inventory for DSM-5 - Brief Form (PID-5-BF). The PID-5-BF (APA, 2013; Zimmermann et al., 2014) is a 25-item measure assessing the broad maladaptive personality domains of negative affectivity, detachment, disinhibition, antagonism, and psychoticism with five items each. Items are rated on a 4-point scale ranging from "very false or often false" (0) to "very true or often true" (3). Internal consistency of the PID-5-BF total score was estimated at $\alpha=.86$ in Sample 2 .

Statistical Analyses. The analyses were performed using R version 4.0.3 (R Core Team, 2020) in conjunction with the package lavaan (Rosseel, 2012) and Mplus version 8.4 (Muthén \& Muthén, 1998-2019). All latent variable models were estimated using the Weighted Least Squares Mean and Variance Adjusted (WLSMV) estimator that was applied to the polychoric correlation matrix. The fit of latent variable models was evaluated by a 
commonly used combination of fit indices and cut-off criteria (i.e., Comparative Fit Index $[\mathrm{CFI}]>$.95, Root Mean Square Error of Approximation $[\mathrm{RMSEA}]<.06$, Standardized Root Mean Square Residual $[\mathrm{SRMR}]<.08 ; \mathrm{Hu} \&$ Bentler, 1999). The internal consistency of the RFQ was estimated with the model-based McDonald's $\omega$ for categorical variables (Flora, 2020). We report fully standardized estimates.

Factor Structure. The factor structure of the RFQ was evaluated by confirmatory factor analysis (CFA) and exploratory factor analysis (EFA) using the original item responses. The two-dimensional measurement model using double-scored items that was reported by the creators of the RFQ (Badoud et al., 2015; Fonagy et al., 2016) is not taken into account for the main results due to the methodological problems associated with the scoring procedure that are described above. However, we report the results based on the original model and the creators' recommendations in the supplement. For this analysis, we considered the following measurement models: (1) a unidimensional CFA; (2) a twodimensional CFA with cross-loadings that follow the scoring procedure of RFQ_C and RFQ_U as proposed in the original publication; and (3) a two-dimensional EFA with oblique factor rotation (quartimin). In all CFA models, the correlation between the residual variances of Items 3 and 4 was freely estimated since these items have a large overlap in terms of semantic content and wording. As two measurement occasions were available in Sample 1 (i.e., at admission and discharge, respectively), we specified repeated measures CFA models with equality constraints for loadings, thresholds, intercepts, latent covariance, and residual covariances (Liu et al., 2017). For the repeated measures CFA, we dealt with missing data by means of pairwise deletion.

U-Shaped Associations with Psychopathology. We investigated the shape of associations between the RFQ scale scores and various measures of psychopathology spanning symptoms of mental illness and maladaptive characteristics. More specifically, 
given the conceptualization of the RFQ, one would expect U-shaped associations with maladaptive characteristics (i.e., signs and symptoms of psychopathology) and inverse Ushaped associations with adaptive characteristics (e.g., subjective well-being), respectively. In order to test the hypothesis that hypo- and hypermentalizing may delineate extreme maladaptive ends of a unidimensional continuum (i.e., very low and very high values on the manifest score), we examined U-shaped and inverse U-shaped associations between the RFQ score and measures of symptomatic distress (i.e., general symptomatic distress: BSI; somatic symptoms: PHQ-15; depressive symptoms vs. well-being: PHQ-9, WHO-5), personality dysfunction (i.e., IPO-16, OPD-SQS, PID-5-BF), and interpersonal distress (i.e., IIP-32). To this end, lowess smoothing curves were inspected and regression models were estimated in which the quadratic term was added to the RFQ score for predicting criterion variables. It should be noted that a significant effect of the quadratic predictor is not sufficiently indicative of the presence of two maladaptive poles, as this would also be observed, for example, in floor or ceiling effects. More specifically, the predicted values for a criterion should also differ between medium scores on the RFQ as compared to extreme scores by forming a Ushape. We therefore further used the two-lines test as a more rigorous method (Simonsohn, 2018) that estimates two regression lines, one before and one after a break-point in the distribution of a predictor variable, in order to detect a change in the sign of the regression slope.

Specific and Latent Associations with Personality Pathology. We investigated the differential associations between the RFQ and indicators of personality pathology (i.e., IPO16, OPD-SQS) and various dimensions of symptomatic distress (i.e., BSI, PHQ-15, PHQ-9, IIP-32, WHO-5), respectively. To this end, we compared the magnitude of their correlation coefficients in Sample 1. Additionally, we estimated the empirical overlap between the RFQ and indicators of personality pathology (i.e., PID-5-BF, IPO-16, OPD-SQS) in both samples 
using a bifactor exploratory structural equation modeling approach (i.e., bifactor ESEM). In a bifactor ESEM (e.g., Morin et al., 2020), a criterion (e.g., RFQ) is regressed on the orthogonal general and specific factors of an exploratory bifactor measurement model reflecting a multidimensional construct (e.g., PID-5-BF). This approach has two advantages for this analysis. First, the use of latent variable modeling partitions reliable variance and measurement error and thus estimates the disattenuated associations among constructs. Second, bifactor models with uncorrelated general and specific factors allow for a clear partitioning of variance in the presence of multidimensionality and a strong general factor (Reise, 2012), as is the case for the PID-5-BF, IPO-16, and OPD-SQS (Zimmermann et al., 2020). Further details and explanations about the bifactor ESEM approach used here can be found in Note S3 of the supplement.

\section{Results}

Factor Structure. All estimated model parameters are depicted in Figure 2 per sample. In Sample 1, the unidimensional repeated measures CFA model showed good model fit, $\chi^{2}(139)=353.0, p<.001, \mathrm{CFI}=.98, \mathrm{RMSEA}=.046,90 \%$ CI $[.041 ; .052]$, SRMR $=.06$. Factor loadings were acceptable $(\lambda \geq .49)$, but Item 7 showed a weak loading on the latent factor $(\lambda=.34)$. The a priori specified residual correlation between Items 3 and 4 was estimated at .60. Heywood cases occurred for the two-dimensional CFA model (i.e., factor correlation $>1$ ) and the two-dimensional EFA model (i.e., standardized factor loading $>1$ for Item 4). In Sample 2, the unidimensional CFA model provided a good fit to the data, $\chi^{2}(19)=$ $91.8, p<.001, \mathrm{CFI}=.98, \mathrm{RMSEA}=.08,90 \% \mathrm{CI}[.07 ; .10], \mathrm{SRMR}=.04$. Factor loadings were acceptable $(\lambda \geq .51)$, but Item 7 again exhibited the weakest loading on the latent factor $(\lambda=.45)$. The a priori specified residual correlation between Items 3 and 4 was estimated at .66. The two-dimensional CFA model did not reach convergence. The two-dimensional EFA model again showed a Heywood case (i.e., standardized loading $>1$ for Item 4). 
Taken together, we did not identify the two proposed dimensions of RFQ_C and RFQ_U in the estimated solutions, and we never identified more than one meaningful factor in general, suggesting that the RFQ essentially captures a unidimensional construct. Results for the originally proposed two-dimensional CFA model that uses double-scoring of RFQ items are reported in the supplement (see Figures S1 and S2). Note that we found similar parameters and model fit for the original specifications based on double-scored items as found in previous validation studies (Badoud et al., 2015; Fonagy et al., 2016). We explained above why these models and their respective fit statistics should rather not be interpreted substantively as they are based on questionable assumptions.

The internal consistency of the RFQ factor from the unidimensional solution was estimated at $\omega=.79 / .81$ (Sample 1; admission/discharge) and $\omega=.82$ (Sample 2), respectively. In the following, we omitted Items 4 and 7 in order to improve the internal consistency of the scale (Spitzer et al., 2021). This decision was based on Item 7 ("I always know what I feel") overall tending towards having low factor loadings, whereas Item 3 ("When I get angry, I say things without really knowing why I am saying them") and Item 4 ("When I get angry, I say things I regret later") overlap strongly with respect to their content. The removal of these two items resulted in a 6-item scale with internal consistency of $\omega=.82$ (Sample 1) and $\omega=.83$ (Sample 2). Subsequent results refer to the 6-item version of the RFQ (i.e., RFQ-6) with low scores supposedly reflecting certainty about mental states and high scores reflecting uncertainty about mental states. ${ }^{2}$ This directionality is a decision based on the circumstance that all items of the RFQ-6 are geared towards an endorsement of uncertainty in their original format.

\footnotetext{
${ }^{2}$ The total scores of the RFQ-8 and the reduced RFQ-6 were correlated at .97 (Sample 1) and at .98 (Sample 2), respectively. It should be emphasized that retaining items 4 and 7 produced highly similar results and equivalent conclusions for all of the presented analyses.
} 
Associations with Psychopathology. We did not find any evidence for (inverse) Ushaped relationships between the RFQ-6 and criteria indicative of symptom distress, personality pathology, or well-being. Although a significant quadratic term was found for OPD-SQS in Sample 1 and for PID-5-BF detachment in Sample 2, the associations were not U-shaped but merely indicated a ceiling effect for higher values of the RFQ-6 (see supplement Figures S3 and S4). The two-lines test did not indicate any U-shaped statistical associations (see Figures S5 and S6). For the unidimensional RFQ-8, U-shaped associations were absent as well.

On the contrary, the RFQ-6 exhibited substantial linear associations with measures of psychopathology (see Tables 1 and 2). In Sample 1, the strongest correlations were found between the RFQ-6 and measures of personality pathology, including the IPO-16 $(r=.72)$ and the OPD-SQS $(r=.65)$, which were also significantly larger $(p<.001$, respectively) than the correlations between the RFQ-6 and other measures, for example as compared to the BSI $(r=.54)$ and the IIP $(r=.54)$. The bivariate associations between the original scales based on double-scoring of the RFQ (i.e., RFQ_C and RFQ_U) as well as the RFQ-8 and criterion measures are supplemented (see Table S1 and Table S2).

In Sample 1, the bifactor ESEM (see Panel A of Figure 3) had acceptable fit, $\chi^{2}(373)$ $=1764.83, p<.001, \mathrm{CFI}=.94, \mathrm{RMSEA}=.07,90 \% \mathrm{CI}[.06 ; .07], \mathrm{SRMR}=.04$. All items of the IPO-16 and OPD-SQS loaded significantly on a general factor of personality pathology with standardized loadings ranging from $\lambda=.32$ to $\lambda=.71$. For the specific factors, target loadings were in the expected direction (i.e., positive factor loadings) and most of them had values of $\lambda>.30$. (Absolute) non-target loadings were consistently $<.30$. The standardized regression coefficient of the general factor was $\beta=.83(p<.001)$, explaining $69 \%$ of variance in the RFQ factor. The specific factors of OPD relationship $(\beta=-.17, p<.001)$, OPD contact $(\beta=.08, p=.005)$, OPD self-perception $(\beta=.14, p<.001)$, IPO reality testing $(\beta=.09, p=$ 
$.001)$, IPO identity diffusion $(\beta=.06, p=.033)$, and IPO primitive defenses $(\beta=.19, p<$ .001 ) incrementally explained a combined $10 \%$ of variance in the RFQ factor. The unexplained variance in the latent RFQ factor thus amounted to $21 \%$. For the (highly similar) results using RFQ-8, see Panel A of Figure S7.

In Sample 2, the bifactor ESEM (see Panel B of Figure 3) had good fit, $\chi^{2}(318)=$ 743.0, $p<.001, \mathrm{CFI}=.97, \mathrm{RMSEA}=.05,90 \% \mathrm{CI}[.04 ; .05], \mathrm{SRMR}=.04$. All items of the PID-5-BF loaded significantly on a general factor of personality pathology with standardized loadings ranging from $\lambda=.31$ to $\lambda=.75$. For the specific factors, target loadings were in the expected direction (i.e., positive factor loadings) and most of them had values of $\lambda>.30$. (Absolute) non-target loadings were consistently $<.30$. The standardized regression coefficient of the general factor was $\beta=.68(p<.001)$, explaining $47 \%$ of variance in the RFQ factor. The specific factors of PID-5-BF negative affect $(\beta=.31, p<.001)$, PID-5-BF detachment $(\beta=.09, p=.022)$, PID-5-BF disinhibition $(\beta=.08, p=.042)$, and PID-5-BF psychoticism $(\beta=.32, p<.001)$ incrementally explained a combined $21 \%$ of variance in the RFQ factor. The unexplained variance in the latent RFQ factor thus amounted to $32 \%$. See Panel B of Figure S7 for the (highly similar) results using all eight RFQ items.

\section{Discussion}

Regarding the factor structure of the RFQ, we found evidence for a unidimensional construct in a large clinical sample (Sample 1) and a large sample of young adults (Sample 2), thereby corroborating initial findings (Spitzer et al., 2021). We have argued that using a unidimensional approach in combination with non-linear statistical modeling is consistent with mentalizing theory and allows for conceptualizing hypo- and hypermentalizing as two maladaptive poles of a continuum. We tested this notion by means of quadratic regression, lowess curves, and the two-lines test. Across a broad range of criterion variables, however, we found no evidence that the RFQ assesses a maladaptive form of having too much certainty 
about mental states (i.e., hypermentalizing). Only the uncertainty pole of the RFQ was associated with poorer mental health. Finally, in line with theoretical expectations with regard to the mentalizing construct (e.g., Bateman \& Fonagy, 2019), the RFQ was more strongly related to indicators of personality dysfunction than to symptomatic distress.

More specifically, we found that the variance of the RFQ primarily reflected broad indicators of self-reported personality dysfunction in both samples (i.e., clinical and nonclinical) using diverse measures (i.e., PID-5-BF, IPO-16, OPD-SQS), whereas comparatively little variance was unique to the RFQ. On the one hand, this may suggest that the constructs of mentalizing and personality pathology, albeit conceptually separate, might be so greatly intertwined that they cannot clearly be distinguished empirically (at least using self-reports). On the other hand, in light of the observation that various items of the RFQ may tap into related maladaptive dispositions (e.g., negative urgency, emotional lability, or impulsivity), the large overlap between the RFQ and dimensions of personality pathology may also signal caution. In fact, this finding could point to potential problems with respect to the discriminant validity of the measure. Consistent with this notion, the existing research literature also provided mixed results for the convergent validity of the RFQ with respect to mentalizing and related constructs. In the initial validation studies, RFQ_U and RFQ_C exhibited strong correlations to alexithymia (comparable to those for indicators of personality pathology) but substantially smaller correlations to cognitive empathy and perspective-taking (Badoud et al., 2015; Fonagy et al., 2016; Morandotti et al., 2018). This might be concerning given that constructs such as cognitive empathy and perspective-taking are very similar to mentalizing by definition (e.g., Ickes, 1993), except that the former constructs solely pertain to understanding others' mental states. In general, additional examinations of the convergence of the RFQ with alternative self-report measures of mentalizing are needed.

\section{Study 2}


To address the question of whether validity issues of the RFQ contribute to its strong empirical overlap with indicators of personality pathology, we conducted a second study that was fully preregistered (see https://osf.io/qr38t). We hypothesized that the item content of the RFQ reflects both mentalizing and possible consequences of impaired mentalizing, namely, emotional lability and impulsivity. If the RFQ conflated these constructs, this could artificially inflate associations between mentalizing as operationalized by the RFQ and indicators of personality dysfunction, thereby impeding the interpretability of RFQ scores and the measure's utility for theory testing. Using commonality analysis (Nimon et al., 2008), we tested whether and to what extent the associations between the RFQ and indicators of personality dysfunction are driven by content overlap rather than reflecting the true association between constructs. Furthermore, we investigated item-level correlations of the RFQ items with convergent and discriminant measures to examine their nomological consistency (Thielmann \& Hilbig, 2019). For example, nomological inconsistency would be indicated by some RFQ items correlating more strongly with impulsivity and other RFQ items correlating more strongly with other measures of mentalizing. Thus, Study 2 included alternative self-report measures of mentalizing, broad measures of personality pathology, and assessments of potential confounders, namely, emotional lability and impulsivity. With regard to alternative measures of mentalizing, we deliberately selected questionnaires that pertain to the core construct of mentalizing, that is, interpreting the mental states of the self and others.

\section{Method}

Sample 3. We recruited participants from the United States online via the panel provider Prolific. Data quality was ensured by a series of attention and validity checks. Participants received minimum wage as compensation. There were no missing data as individuals were not able to proceed without answering each item. We collected data from $N$ 
$=862$ participants based on an a priori power analysis (see preregistration). Participants' (47\% female) age ranged from 18 to $75(M=34.9, S D=11.7)$. For more details about exclusion criteria and sample characteristics, see Note S4.

Measures. In the study, all questionnaires were presented in random order. The RFQ (Fonagy et al., 2016) and the IPO-16 (Zimmermann et al., 2013) were administered again in their respective English versions. Based on our findings from Study 1, we used a unidimensional mean score of the RFQ such that high values reflected uncertainty about mental states. The analyses were performed for both the RFQ-8 and the RFQ- 6 . The internal consistencies of the RFQ-6 $(\omega=.87)$, RFQ-8 $(\omega=.87)$, and the IPO-16 $(\alpha=.90)$ were good.

Level of Personality Functioning Scale - Brief Form 2.0 (LPFS-BF). The LPFS-BF (Weekers et al., 2019) is a 12-item self-report measure assessing impairments in the domains of self-functioning (6 items) and interpersonal functioning (6 items). The items (e.g., "I often make unrealistic demands on myself") are answered on a 4-point scale ranging from "completely untrue" (1) to "completely true" (4). High scores on the respective scales indicate self-dysfunction or interpersonal dysfunction. Internal consistency of the LPFS-BF total score was estimated at $\alpha=.90$.

Certainty About Mental States Questionnaire (CAMSQ). The CAMSQ (Müller et al., 2021 ) is a 20 -item self-report measure of mentalizing that assesses the perceived certainty associated with making inferences about the mental states of the self (i.e., Self-Certainty) and others (i.e., Other-Certainty). The items capture affective, cognitive, and motivational content (e.g., "I understand my feelings", "I know when other people are hiding their thoughts") and are answered on a 7-point frequency scale ranging from "never" (1) to "always" (7). High scores reflect high levels of certainty. Internal consistencies of CAMSQ Self-Certainty ( $\alpha=$ $.93)$ and CAMSQ Other-Certainty $(\alpha=.92)$ were high. 
Empathy Quotient (EQ). The EQ (Baron-Cohen \& Wheelwright, 2004) is a 40-item self-report measure of empathy. The 9-item Cognitive Empathy scale was used as a measure of mentalizing others. Items (e.g., "I am good at predicting how someone will feel”) are rated on a 4-point scale ranging from "strongly disagree" (1) to "strongly agree" (4). Internal consistency of the EQ Cognitive Empathy scale was estimated at $\alpha=.90$.

Self-Reflection and Insight Scale (SRIS). The SRIS (Grant et al., 2002) is a 20-item self-report measure. The 8-item Self-Insight scale of the SRIS was used as a measure of mentalizing oneself. Items (e.g., "I usually know why I feel the way I do") are rated on a 6point scale ranging from "strongly disagree" (1) to "strongly agree" (6). Internal consistency of the SRIS Self-Insight scale was estimated at $\alpha=.88$.

UPPS-P Impulsive Behavior Scale (UPPS-P). The UPPS-P (Lynam et al., 2007) assesses five impulsive personality traits with 59 items. We used the 12 -item Negative Urgency scale that assesses impulsivity in terms of acting rashly under the influence of negative emotions. Items (e.g., "When I am upset, I often act without thinking”) are endorsed or rejected on a 4-point scale ranging from "disagree strongly" (1) to "agree strongly" (4). Internal consistency of the UPPS-P Negative Urgency scale was estimated at $\alpha=.93$.

Difficulties in Emotion Regulation Scale (DERS). The DERS (Gratz \& Roemer, 2004) is a 36-item self-report measure that assesses various aspects of emotional dysregulation. We used the 6-item Impulse Control Difficulties subscale to assess impulsivity. Items (e.g., "When I'm upset, I become out of control”) are rated on a 5-point scale ranging from "almost never" (1) to "almost always" (5) indicating the frequency of experiencing the described behavior. Internal consistency of the DERS Impulse Control Difficulties scale was $\alpha=.90$.

Personality Inventory for DSM-5 (PID-5). The PID-5 (APA, 2013) assesses personality pathology according to the AMPD with 220 items. The 7-item Emotional Lability 
facet scale was used. Items (e.g., "I am a highly emotional person") are answered on a 4-point scale ranging from "very false or often false" (0) to "very true or often true" (3). Internal consistency of the PID-5 Emotional Lability scale was $\alpha=.91$.

Personality Assessment Inventory - Borderline Features (PAI-BOR). The PAI-BOR (Morey, 2004) assesses traits associated with borderline personality disorder using 24 items. We used the six items of the Affective Instability scale to assess emotional lability. Items (e.g., "My mood can shift quite suddenly") are rated on a 4-point scale ranging from "false, not at all true" (0) to "very true" (3). Internal consistency of the PAI-BOR Affective Instability scale was $\alpha=.80$.

Statistical Analysis. To test the hypothesis of whether the associations between the RFQ and indicators of personality pathology are driven by content overlap, we conducted a commonality analysis (Nimon et al., 2008) that estimates the common and unique contributions of each predictor in predicting a criterion. Commonality analysis involves estimating a series of multiple regression models considering all possible combinations of predictors. Specifically, the RFQ mean score is regressed on a broad measure of personality dysfunction, other measures of mentalizing the self and others, and measures of emotional lability and impulsivity. We expected that the effect of personality dysfunction on the RFQ mean score can be partly attributed to variation that both measures share with mentalizing impairment, while another significant part can be attributed to variation shared with emotional lability and impulsivity alone (i.e., variation that is non-overlapping with other measures of mentalizing). For example, the manifest correlation between the RFQ mean score and indicators of personality dysfunction amounted to an $r$ of around .65 in Study 1, corresponding to a shared variance of $R^{2}=.42$. Using commonality analysis, we decomposed the variance explained in the RFQ by the respective measure of personality dysfunction into (a) variance that is shared with the respective measures of impulsivity and emotional lability 
but not with the respective measures of mentalizing the self and others, (b) variance that is shared with the respective measures of mentalizing the self and others, and (c) variance explained uniquely by the respective measure of personality dysfunction. We performed a permutation test using 5000 random permutations of the dependent variable to compute $p$ values for testing the null hypothesis that part (a) equals zero (i.e., that no part of the variance explained is due to variance shared with emotional lability and impulsivity alone). We tested this hypothesis with two different sets of measures to facilitate the generalizability of the results (irrespective of specific measures; see preregistration). To control for performing this test twice for each set of variables, we considered a $p$-value less than .025 to be indicative of statistical significance. Furthermore, we computed bias-corrected bootstrap confidence intervals for evaluating the precision of the estimate using 5000 bootstrap resamples. The commonality analysis was conducted via the R package yhat (Nimon et al., 2021).

We also examined the pattern of associations between the eight RFQ items and personality dysfunction, emotional lability, impulsivity, and other measures of mentalizing to evaluate whether these correlations differ in magnitude. Differential patterns of association would indicate that the eight RFQ items are not nomologically consistent (Thielmann \& Hilbig, 2019). For the sake of completeness, we again tested the factor models considered in Study 1 .

\section{Results}

We provide open data and a script for reproducing the analyses in $\mathrm{R}$ (https://osf.io/stbz5). As in Study 1, the unidimensional CFA model fit the data well, $\chi^{2}(19)=$ $272.7, p<.001, \mathrm{CFI}=.98, \mathrm{RMSEA}=.125,90 \% \mathrm{CI}[.112 ; .138], \mathrm{SRMR}=.04$, whereas twodimensional models did not provide parameter estimates in line with the proposed structure of RFQ_C and RFQ_U (see Figure 2). Measures that were intended to assess the same constructs (e.g., CAMSQ Self-Certainty and SRIS Self-Insight) showed the expected high 
convergent correlations. The RFQ mean score exhibited similarly strong associations with broad indicators of personality dysfunction, measures of emotional lability and impulsivity, and measures of mentalizing the self (see Table 3 and Table S3 for the full correlation matrices including RFQ-6 and RFQ-8). Rather low correlations were observed between the RFQ mean score and measures of mentalizing others, indicating that the RFQ primarily pertains to mentalizing the self.

Following our preregistration, we used commonality analysis to estimate what content (i.e., mentalizing, emotional lability, impulsivity) accounts for the association between the RFQ and indicators of personality dysfunction. Using the first set of variables, the association between the RFQ-6 and LPFS-BF $\left(R^{2}=.46\right)$ was decomposed into variance shared with measures of mentalizing $\left(\Delta R^{2}=.22,95 \% \mathrm{CI}[.14 ; .27], p<.001\right)$, variance shared with measures of emotional lability and impulsivity alone $\left(\Delta R^{2}=.20,95 \%\right.$ CI $\left.[.16 ; .30], p<.001\right)$, and variance uniquely explained by LPFS-BF $\left(\Delta R^{2}=.04,95 \%\right.$ CI $\left.[.02 ; .06], p<.001\right)$. In the second set of variables, the association between the RFQ-6 and IPO-16 $\left(R^{2}=.45\right)$ was decomposed into variance shared with measures of mentalizing $\left(\Delta R^{2}=.33,95 \% \mathrm{CI}[.24 ; .38]\right.$, $p<.001)$, variance shared with measures of emotional lability and impulsivity alone $\left(\Delta R^{2}=\right.$ $.07,95 \%$ CI $[.04 ; .18], p<.001)$, and variance uniquely explained by IPO- $16\left(\Delta R^{2}=.05,95 \%\right.$ CI $[.03 ; .07], p<.001)$. In light of the difference between the two arbitrarily selected sets of variables, we then conducted the commonality analysis across all 32 possible variable combinations to account for influences of specific combinations. On average, the association between the RFQ-6 and broad indicators of personality dysfunction (mean $R^{2}=.45$ ) was decomposed into variance shared with measures of mentalizing (mean $\Delta R^{2}=.27$ ), variance shared with measures of emotional lability and impulsivity alone (mean $\Delta R^{2}=.14$ ), and variance uniquely explained by measures of personality dysfunction (mean $\Delta R^{2}=.04$ ). Thus, $59 \%$ of the observed associations between RFQ- 6 and indicators of personality dysfunction 
were due to variance shared with other measures of mentalizing, whereas $31 \%$ were due to variance shared with measures of emotional lability and impulsivity alone, and $10 \%$ were unique to measures of personality dysfunction. Very similar results were obtained using the RFQ-8 (see Note S5).

The item-level analysis demonstrated nomological inconsistencies between the eight items of the RFQ in terms of significant differences in their correlational patterns (see Table 4). Generally, the item-level associations were most pronounced for UPPS-P Negative Urgency as a measure of impulsivity and for SRIS Self-Insight as a measure of mentalizing the self. Thus, we specifically used these two scales for testing differences in the magnitude of correlations, although it should be noted that the correlational patterns were consistent for the other measures that were included. RFQ Items 3, 4, 5, and 8 correlated significantly stronger $(p<.001)$ with Negative Urgency than with Self-Insight. In contrast, Items 1,2 , and 7 correlated significantly stronger with Self-Insight than with Negative Urgency $(p<.001)$.

\section{Discussion}

In Study 2, we aimed to test the hypothesis that the items of the RFQ conflate content associated with mentalizing and content associated with assumed consequences of mentalizing impairment (i.e., emotional lability and impulsivity). The preregistered analyses provide evidence for this hypothesis and suggest that associations between the RFQ and measures of personality dysfunction may be inflated by approximately $30 \%$ because they exploit common variance with aspects of emotional lability and impulsivity. These results point to limitations of the RFQ with regard to its convergent and discriminant validity. Specifically, the item-level analysis indicated nomological inconsistencies (Thielmann \& Hilbig, 2019) that suggest RFQ Item 3 ("When I get angry I say things without really knowing why I am saying them"), Item 4 ("When I get angry, I say things that I later regret"), Item 5 ("If I feel insecure I can behave in ways that put others' backs up"), and Item 8 
("Strong feelings often cloud my thinking") may be the reason for the conflation because they converge with negative urgency rather than mentalizing. Study 2 further illustrates the relevance of assessing mentalizing the self and mentalizing others separately, as these two dimensions provide unique information. Considering that the RFQ only contains one item that is clearly geared towards understanding others' mental states (i.e., Item 1: "People's thoughts are a mystery to me"), the RFQ cannot measure mentalizing others with sufficient fidelity.

\section{General Discussion}

The RFQ has been proposed as a short self-report measure of reflective functioning. In this article, we have elaborated our concerns with respect to the instrument's validity and the methodology used in prior studies (e.g., Badoud et al., 2015; Fonagy et al., 2016), particularly in reference to its item content, scoring procedure, dimensionality, and associations with psychopathology. Using large clinical and non-clinical samples from Germany and the US, we augmented the critical discussion with new empirical analyses. First, our findings suggest that the RFQ assesses a unidimensional construct. Consequently, we recommend refraining from the originally proposed scoring procedure to derive RFQ_C and RFQ_U (Fonagy et al., 2016) and instead relying on a unidimensional score using the original responses to the RFQ items (such as mean scores on the psychometrically optimized RFQ-6 or the RFQ-8). Second, we have demonstrated that although the RFQ reflects mentalizing impairments regarding the self, it also exhibits a substantial confound with emotional lability and impulsivity due to shared item content. This indicates that, when using the RFQ to study research questions related to mentalizing theory, observed associations with other constructs can be influenced by this confound and inferences can thus be impeded. Third, consistent with previous accounts (e.g., de Meulemeester et al., 2018), the present results further indicate that the RFQ does indeed capture a maladaptive form of having too 
little certainty about mental states (i.e., hypomentalizing) but appears to be unable to capture a maladaptive form of having too much certainty about mental states (i.e., hypermentalizing) as its certainty pole does not exhibit positive associations with negative outcomes. One reason for this could be that the RFQ does not measure variation in hypermentalizing with sufficient reliability on the certainty pole of the continuum (e.g., because its items are formulated with reference to uncertainty).

It should be noted that the present empirical findings are limited to the English and the German versions of the RFQ and might not necessarily generalize to other languages. However, our concerns about the item content and the scoring procedure apply to the various translations as well. Moreover, our conclusions cannot be generalized to the long forms of the RFQ (e.g., Euler et al., 2021), although it should be noted that, to date, no validation studies have been published for these forms and they have been seldomly used in previous research. Finally, the current study is subject to the limitation that it did not focus on the primary target population that is assumed to show particularly severe impairments in mentalizing capacity, that is, individuals with borderline personality pathology (Luyten et al., 2020).

Mentalizing is arguably an important psychological construct with great relevance for psychopathology, personality, and psychotherapy research (APA, 2013; Bender et al., 2011). This calls for a valid and economic self-report assessment of the construct. Although the RFQ has been rather broadly accepted by the field and is used in a growing body of research, we have argued that the validity evidence is not compelling. In our view, self-report assessments of mentalizing should adhere more closely to the specific core of the construct (i.e., inferring mental states) rather than emphasizing hypothetical consequences of impaired mentalizing (e.g., impulsivity). Thereby, the conflation of different constructs could be avoided. Second, both hypo- and hypermentalizing should ideally be captured by measures of mentalizing. Indeed, demonstrating that these two maladaptive variants of mentalizing impairment can be 
assessed by self-report questionnaires is an important empirical test in itself that would increase confidence in construct validity. For example, the CAMSQ (Müller et al., 2021) was recently introduced as a measure that focuses on the core definition of inferring mental states of the self and others provided by Fonagy and colleagues (2016). Initial results for the CAMSQ suggest that it captures maladaptive levels of both too little or too much certainty about mental states that could be interpreted as forms of hypo- and hypermentalizing.

\section{Conclusion}

The RFQ is regularly used to study the concept of mentalizing. Herein, we have outlined critical considerations regarding the validity of the RFQ and provided empirical evidence to support the critique. Findings indicate that the RFQ is a unidimensional measure that reflects hypomentalizing pertaining to the self but is also conflated with content related to emotional lability and impulsivity. Thus, researchers and mental health professionals alike should be rather cautious in using the RFQ for theory testing and individual assessment. 


\section{References}

American Psychiatric Association. (2013). Diagnostic and statistical manual of mental disorders (DSM-5®). American Psychiatric Pub.

Back, S., Zettl, M., Bertsch, K., \& Taubner, S. (2020). Persönlichkeitsniveau, maladaptive Traits und Kindheitstraumata. Psychotherapeut, 65, 374-382. https://doi.org/10.1007/s00278-020-00445-7

Badoud, D., Luyten, P., Fonseca-Pedrero, E., Eliez, S., Fonagy, P., \& Debbané, M. (2015). The French version of the Reflective Functioning Questionnaire: Validity data for adolescents and adults and its association with non-suicidal self-injury. PloS ONE, 10, e0145892. https://doi.org/10.1371/journal.pone.0145892

Badoud, D., Prada, P., Nicastro, R., Germond, C., Luyten, P., Perroud, N., \& Debbané, M. (2018). Attachment and reflective functioning in women with borderline personality disorder. Journal of Personality Disorders, 32, 17-30. https://doi.org/10.1521/pedi_2017 $31 \quad 283$

Baron-Cohen, S., \& Wheelwright, S. (2004). The empathy quotient: An investigation of adults with Asperger syndrome or high functioning autism, and normal sex differences. Journal of Autism and Developmental Disorders, 34, 163-175. https://doi.org/10.1023/b:jadd.0000022607.19833.00

Bateman, A., \& Fonagy, P. (2016). Mentalization-based treatment for personality disorders: A practical guide. Oxford, UK: Oxford Univ. Press.

Bateman, A. W., \& Fonagy, P. (Eds.). (2019). Handbook of mentalizing in mental health practice. American Psychiatric Publishing, Inc.

Bender, D. S., Morey, L. C., \& Skodol, A. E. (2011). Toward a model for assessing level of personality functioning in DSM-5, part I: A review of theory and methods. Journal of Personality Assessment, 93, 332-346. https://doi.org/10.1080/00223891.2011.583808 
Carver, C. S., Johnson, S. L., \& Timpano, K. R. (2017). Toward a functional view of the $p$ factor in psychopathology. Clinical Psychological Science, 5, 880-889. https://doi.org/10.1177/2167702617710037

Cyders, M. A., \& Smith, G. T. (2008). Emotion-based dispositions to rash action: Positive and negative urgency. Psychological Bulletin, 134, 807-828. https://doi.org/10.1037/a0013341

de Meulemeester, C., Lowyck, B., Vermote, R., Verhaest, Y., \& Luyten, P. (2017). Mentalizing and interpersonal problems in borderline personality disorder: The mediating role of identity diffusion. Psychiatry Research, 258, 141-144. https://doi.org/10.1016/j.psychres.2017.09.061

de Meulemeester, C., Vansteelandt, K., Luyten, P., \& Lowyck, B. (2018). Mentalizing as a mechanism of change in the treatment of patients with borderline personality disorder: A parallel process growth modeling approach. Personality Disorders: Theory, Research, and Treatment, 9, 22-29. https://doi.org/10.1037/per0000256

Ehrenthal, J. C., Dinger, U., Schauenburg, H., Horsch, L., Dahlbender, R. W., \& Gierk, B. (2015). Entwicklung einer Zwölf-Item-Version des OPD-Strukturfragebogens (OPDSFK). Zeitschrift für Psychosomatische Medizin und Psychotherapie, 61, 262-274. https://doi.org/10.13109/zptm.2015.61.3.262

Euler, S., Nolte, T., Constantinou, M., Griem, J., Montague, P. R., Fonagy, P., \& Personality and Mood Disorders Research Network. (2021). Interpersonal problems in borderline personality disorder: Associations with mentalizing, emotion regulation, and impulsiveness. Journal of Personality Disorders, 35, 177-193. https://doi.org/10.1521/pedi_2019 $33 \quad 427$

Flora, D. B. (2020). Your coefficient alpha is probably wrong, but which coefficient omega is right? A tutorial on using R to obtain better reliability estimates. Advances in Methods 
and Practices in Psychological Science, 3, 484-501.

https://doi.org/10.1177/2515245920951747

Fonagy, P., Luyten, P., Allison, E., \& Campbell, C. (2017). What we have changed our minds about: Part 1. Borderline personality disorder as a limitation of resilience. Borderline Personality Disorder and Emotion Dysregulation, 4, 1-11. https://doi.org/10.1186/s40479-017-0061-9

Fonagy, P., Luyten, P., Moulton-Perkins, A., Lee, Y.-W., Warren, F., Howard, S., Ghinai, R., Fearon, P., \& Lowyck, B. (2016). Development and validation of a self-report measure of mentalizing: The Reflective Functioning Questionnaire. PLoS ONE, 11, e0158678. https://doi.org/10.1371/journal.pone.0158678

Franke, H. (2000). The Brief Symptom Inventory - Deutsche Version. Manual. Göttingen: Beltz.

Grant, A. M., Franklin, J., \& Langford, P. (2002). The self-reflection and insight scale: A new measure of private self-consciousness. Social Behavior and Personality: An International Journal, 30, 821-835. https://doi.org/10.2224/sbp.2002.30.8.821

Gratz, K. L., \& Roemer, L. (2004). Multidimensional assessment of emotion regulation and dysregulation: Development, factor structure, and initial validation of the difficulties in emotion regulation scale. Journal of Psychopathology and Behavioral Assessment, 26, 41-54. https://doi.org/10.1023/B:JOBA.0000007455.08539.94

Horowitz, L. M., Alden, L. E., Kordy, H., \& Strauß, B. (2000). Inventar zur Erfassung interpersonaler Probleme: Deutsche Version; IIP-D. Beltz-Test.

Hu, L. T., \& Bentler, P. M. (1999). Cutoff criteria for fit indexes in covariance structure analysis: Conventional criteria versus new alternatives. Structural Equation Modeling: A Multidisciplinary Journal, 6, 1-55. https://doi.org/10.1080/10705519909540118 
Huang, Y. L., Fonagy, P., Feigenbaum, J., Montague, P. R., Nolte, T., \& Mood Disorder Research Consortium. (2020). Multidirectional pathways between attachment, mentalizing, and posttraumatic stress symptomatology in the context of childhood trauma. Psychopathology, 53, 48-58. https://doi.org/10.1159/000506406

Ickes, W. (1993). Empathic accuracy. Journal of Personality, 61, 587-610. https://doi.org/10.1111/j.1467-6494.1993.tb00783.x

King, K. M., Feil, M. C., \& Halvorson, M. A. (2018). Negative urgency is correlated with the use of reflexive and disengagement emotion regulation strategies. Clinical Psychological Science, 6, 822-834. https://doi.org/10.1177/2167702618785619

Kroenke, K., Spitzer, R. L., \& Williams, J. B. (2001). The PHQ-9: Validity of a brief depression severity measure. Journal of General Internal Medicine, 16, 606-613. https://doi.org/10.1046/j.1525-1497.2001.016009606.x

Kroenke, K., Spitzer, R. L., \& Williams, J. B. (2002). The PHQ-15: Validity of a new measure for evaluating the severity of somatic symptoms. Psychosomatic Medicine, $64,258-266$.

Li, E. T., Carracher, E., \& Bird, T. (2020). Linking childhood emotional abuse and adult depressive symptoms: The role of mentalizing incapacity. Child Abuse \& Neglect, 99, 104253. https://doi.org/10.1016/j.chiabu.2019.104253

Liu, Y., Millsap, R. E., West, S. G., Tein, J. Y., Tanaka, R., \& Grimm, K. J. (2017). Testing measurement invariance in longitudinal data with ordered-categorical measures. Psychological Methods, 22, 486-506. https://psycnet.apa.org/doi/10.1037/met0000075

Luyten, P., Campbell, C., Allison, E., \& Fonagy, P. (2020). The mentalizing approach to psychopathology: State of the art and future directions. Annual Review of Clinical Psychology, 16, 297-325. https://doi.org/10.1146/annurev-clinpsy-071919-015355 
Lynam, D. R., Smith, G. T., Cyders, M. A., Fischer, S., \& Whiteside, S. P. (2007). The UPPS-P questionnaire measure of five dispositions to rash action. Unpublished technical report, Purdue University.

Morandotti, N., Brondino, N., Merelli, A., Boldrini, A., De Vidovich, G. Z., Ricciardo, S., Abbiati, V., Ambrosi, P., Cavercasi, E., Fonagy, P., \& Luyten, P. (2018). The Italian version of the Reflective Functioning Questionnaire: Validity data for adults and its association with severity of borderline personality disorder. PloS ONE, 13, e0206433. https://doi.org/10.1371/journal.pone.0206433

Morey, L. C. (2004). The Personality Assessment Inventory (PAI). Lawrence Erlbaum Associates Publishers.

Morin, A. J., Myers, N. D., \& Lee, S. (2020). Modern Factor Analytic Techniques: Bifactor Models, Exploratory Structural Equation Modeling (ESEM), and Bifactor-ESEM. Handbook of Sport Psychology, 51, 1044-1073. https://doi.org/10.1002/9781119568124.ch51

Müller, S., Wendt, L. P., \& Zimmermann, J. (2021, May 19). Development and Validation of the Certainty About Mental States Questionnaire (CAMSQ): A Self-Report Measure of Mentalizing Oneself and Others. https://doi.org/10.31234/osf.io/jtc3s

Muthén, L. K., \& Muthén, B. O. (1998-2019). Mplus User's Guide. $8^{\text {th }}$ Edition. Los Angeles, CA: Muthén \& Muthén.

Nimon, K., Lewis, M., Kane, R., \& Haynes, R. M. (2008). An R package to compute commonality coefficients in the multiple regression case: An introduction to the package and a practical example. Behavior Research Methods, 40, 457-466. https://doi.org/10.3758/BRM.40.2.457

Nimon, K., Oswald, F., \& Roberts, J. K. (2021). yhat: Interpreting Regression Effects. R package version 2.0-3. https://CRAN.R-project.org/package=yhat 
R Core Team. (2020). R: A language and environment for statistical computing. $\mathrm{R}$ Foundation for Statistical Computing, Vienna, Austria.

Reise, S. P. (2012). The rediscovery of bifactor measurement models. Multivariate Behavioral Research, 47, 667-696. https://doi.org/10.1080/00273171.2012.715555

Rosseel, Y. (2012). lavaan: An R package for structural equation modeling. Journal of Statistical Software, 48, 1-36. http://www.jstatsoft.org/v48/i02/

Settles, R. E., Fischer, S., Cyders, M. A., Combs, J. L., Gunn, R. L., \& Smith, G. T. (2012). Negative urgency: A personality predictor of externalizing behavior characterized by neuroticism, low conscientiousness, and disagreeableness. Journal of Abnormal Psychology, 121, 160-172. https://doi.org/10.1037/a0024948

Simonsohn, U. (2018). Two lines: A valid alternative to the invalid testing of U-shaped relationships with quadratic regressions. Advances in Methods and Practices in Psychological Science, 1, 538-555. https://doi.org/10.1177/2515245918805755

Spitzer, C., Zimmermann, J., Brähler, E., Euler, S., Wendt, L. P., \& Müller, S. (2021). Die deutsche Version des Reflective Functioning Questionnaire (RFQ): Eine teststatistische Überprüfung in der Allgemeinbevölkerung. Psychotherapie Psychosomatik - Medizinische Psychologie, 71, 124-131. https://doi.org/10.1055/a$\underline{1234-6317}$

Thielmann, I., \& Hilbig, B. E. (2019). Nomological consistency: A comprehensive test of the equivalence of different trait indicators for the same constructs. Journal of Personality, 87, 715-730. https://doi.org/10.1111/jopy.12428

Weekers, L. C., Hutsebaut, J., \& Kamphuis, J. H. (2019). The Level of Personality Functioning Scale-Brief Form 2.0: Update of a brief instrument for assessing level of personality functioning. Personality and Mental Health, 13, 3-14. https://doi.org/10.1002/pmh.1434 
World Health Organization. (1998). Wellbeing measures in primary health care / The Depcare Project. WHO Regional Office for Europe: Copenhagen.

Zimmermann, J., Altenstein, D., Krieger, T., Grosse Holtforth, M., Pretsch, J., Alexopoulos, J., Spitzer, C., Benecke, C., Krueger, R. F., Markon, K. E., \& Leising, D. (2014). The structure and correlates of self-reported DSM-5 maladaptive personality traits:

Findings from two German-speaking samples. Journal of Personality Disorders, 28, 518-540. https://doi.org/10.1521/pedi $2014 \quad 28 \quad 130$

Zimmermann, J., Benecke, C., Hörz, S., Rentrop, M., Peham, D., Bock, A., Wallner, T., Schauenburg, H., Frommer, J., Huber, D., Clarkin, J. F., \& Dammann, G. (2013). Validierung einer deutschsprachigen 16-Item-Version des Inventars der Persönlichkeitsorganisation (IPO-16). Diagnostica, 59, 3-16. https://doi.org/10.1026/0012-1924/a000076

Zimmermann, J., Müller, S., Bach, B., Hutsebaut, J., Hummelen, B., \& Fischer, F. (2020). A common metric for self-reported severity of personality disorder. Psychopathology, 53, 161-171. https://doi.org/10.1159/000507377 
Table 1

Bivariate Correlations in Sample 1 at Admission

\begin{tabular}{lccccccc}
\hline & RFQ-6 & BSI & PHQ-15 & PHQ-9 & IIP-32 & IPO-16 & OPD-SQS \\
\hline BSI & .54 & & & & & & \\
PHQ-15 & .26 & .59 & & & & & \\
PHQ-9 & .41 & .76 & .47 & & & & \\
IIP-32 & .54 & .63 & .32 & .51 & & \\
IPO-16 & .72 & .59 & .33 & .46 & .60 & & \\
OPD-SQS & .65 & .72 & .41 & .59 & .68 & .69 & \\
WHO-5 & -.22 & -.51 & -.29 & -.65 & -.35 & -.22 & -.38 \\
\hline
\end{tabular}

Note. $N=861$. All correlations are statistically significant at $p<.001$. 
Table 2

Bivariate Correlations in Sample 2

\begin{tabular}{lcccccc}
\hline & RFQ-6 & Total & NEG & DET & ANT & DIS \\
\hline PID-5-BF Total Score & .68 & & & & & \\
Negative Affectivity (NEG) & .58 & .73 & & & & \\
Detachment (DET) & .47 & .72 & .43 & & & \\
Antagonism (ANT) & .32 & .65 & .29 & .29 & & \\
Disinhibition (DIS) & .45 & .73 & .40 & .36 & .43 & \\
Psychoticism (PSY) & .63 & .82 & .52 & .52 & .44 & .50 \\
\hline
\end{tabular}

Note. $N=566$. All correlations are statistically significant at $p<.001$. 


\section{Table 3}

Bivariate Correlations in Sample 3

\begin{tabular}{|c|c|c|c|c|c|c|c|c|c|c|c|}
\hline & & RFQ-6 & $(1)$ & $(2)$ & (3) & $(4)$ & (5) & $(6)$ & (7) & $(8)$ & (9) \\
\hline (1) & LPFS-BF & .68 & & & & & & & & & \\
\hline$(2)$ & IPO-16 & .67 & .66 & & & & & & & & \\
\hline (3) & CAMSQ Self-Certainty & -.50 & -.51 & -.38 & & & & & & & \\
\hline (4) & SRIS Self-Insight & -.67 & -.60 & -.60 & .68 & & & & & & \\
\hline$(5)$ & CAMSQ Other-Certainty & -.25 & -.23 & -.09 & .56 & .28 & & & & & \\
\hline$(6)$ & EQ Cognitive Empathy & -.26 & -.27 & -.11 & .42 & .28 & .73 & & & & \\
\hline (7) & PID-5 Emotional Lability & .60 & .59 & .61 & -.36 & -.53 & -.08 & -.08 & & & \\
\hline$(8)$ & PAI-BOR Affective Instability & .61 & .67 & .56 & -.42 & -.53 & -.16 & -.17 & .72 & & \\
\hline (9) & UPPS-P Negative Urgency & .71 & .63 & .59 & -.43 & -.54 & -.18 & -.16 & .62 & .69 & \\
\hline$(10)$ & DERS Impulse Control Difficulties & .63 & .65 & .61 & -.41 & -.57 & -.13 & -.15 & .66 & .69 & .68 \\
\hline
\end{tabular}

Note. $N=862$. All correlations are statistically significant at $p<.05$. 


\section{Table 4}

Bivariate Correlations Between the Items of the RFQ and Further Measures in Sample 3

\begin{tabular}{|c|c|c|c|c|c|c|c|c|c|}
\hline & & \multicolumn{8}{|c|}{ RFQ-8 } \\
\hline & & Item 1 & Item 2 & Item 3 & Item 4 & Item 5 & Item 6 & Item 7 & Item 8 \\
\hline (1) & LPFS-BF & .40 & .60 & .51 & .43 & .45 & .59 & .41 & .52 \\
\hline$(2)$ & IPO-16 & .39 & .54 & .51 & .42 & .48 & .57 & .22 & .54 \\
\hline (3) & CAMSQ Self-Certainty & -.27 & -.50 & -.38 & -.29 & -.31 & -.49 & -.58 & -.38 \\
\hline (4) & SRIS Self-Insight & -.41 & -.65 & -.50 & -.39 & -.42 & -.62 & -.51 & -.52 \\
\hline$(5)$ & CAMSQ Other-Certainty & -.31 & -.23 & -.15 & -.17 & -.11 & -.22 & -.30 & -.16 \\
\hline$(6)$ & EQ Cognitive Empathy & -.36 & -.25 & -.15 & -.14 & -.11 & -.23 & -.30 & -.12 \\
\hline (7) & PID-5 Emotional Lability & .26 & .47 & .48 & .40 & .42 & .49 & .24 & .56 \\
\hline$(8)$ & PAI-BOR Affective Instability & .24 & .50 & .50 & .50 & .41 & .53 & .34 & .54 \\
\hline$(9)$ & UPPS-P Negative Urgency & .25 & .56 & .66 & .68 & .51 & .62 & .31 & .59 \\
\hline (10) & DERS Impulse Control Difficulties & .29 & .50 & .58 & .51 & .41 & .54 & .30 & .52 \\
\hline
\end{tabular}

Note. $N=862$. All correlations are statistically significant at $p<.001$. The strongest correlation for each RFQ item on a descriptive level is highlighted in bold. 


\section{Figure 1}

Empirical Distributions of Item 6 Before and After Applying the Scoring Procedure Outlined in Fonagy et al. (2016)
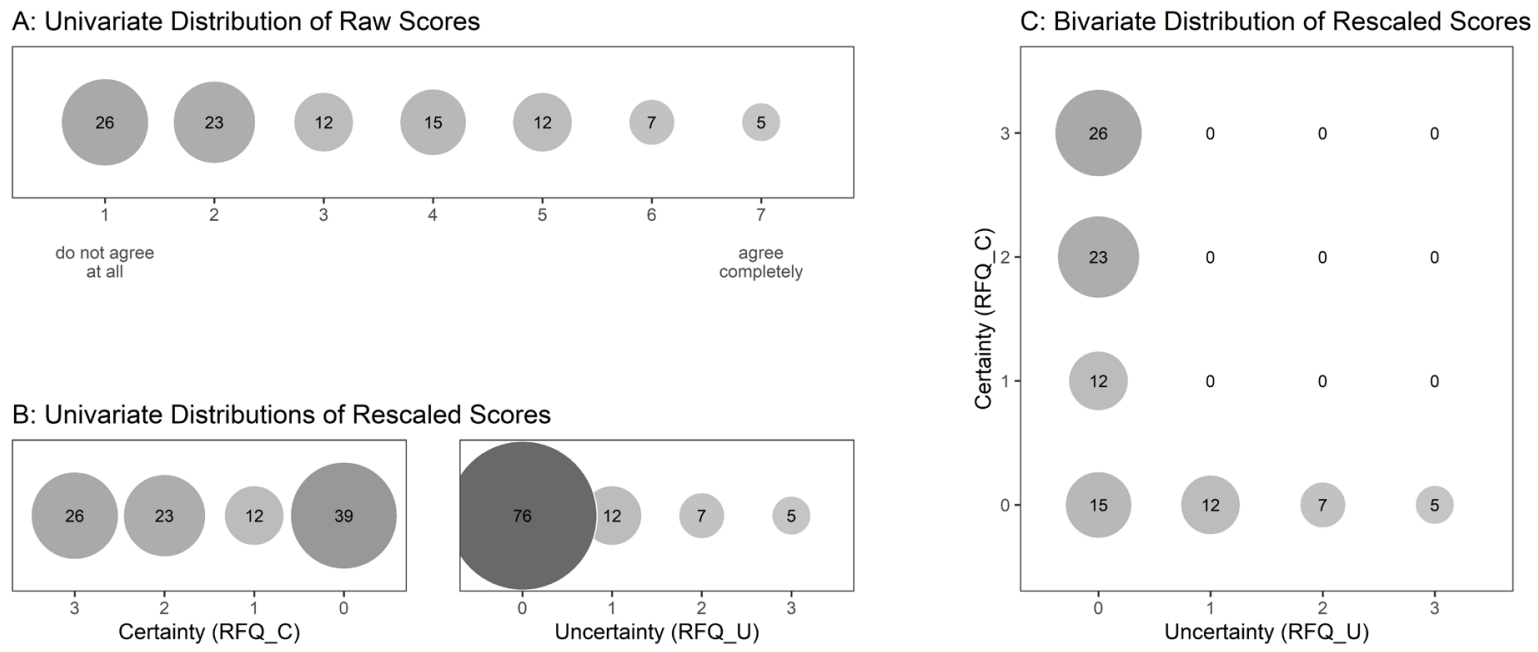

Note. Relative frequencies (in \%) of responses to Item 6 ("Sometimes I do things without really knowing why") in Sample 1. Panel A shows the univariate distribution of raw scores on the 7-point scale, Panel B shows the univariate distributions of the rescaled scores on the 4point scales, and Panel C shows the bivariate distribution of the rescaled scores. 


\section{Figure 2}

Standardized parameter estimates of the factor models in all samples of Study 1 (Samples 1 and 2) and Study 2 (Sample 3)

Clinical Sample 1 (GER)

Non-Clinical Sample 3 (US)
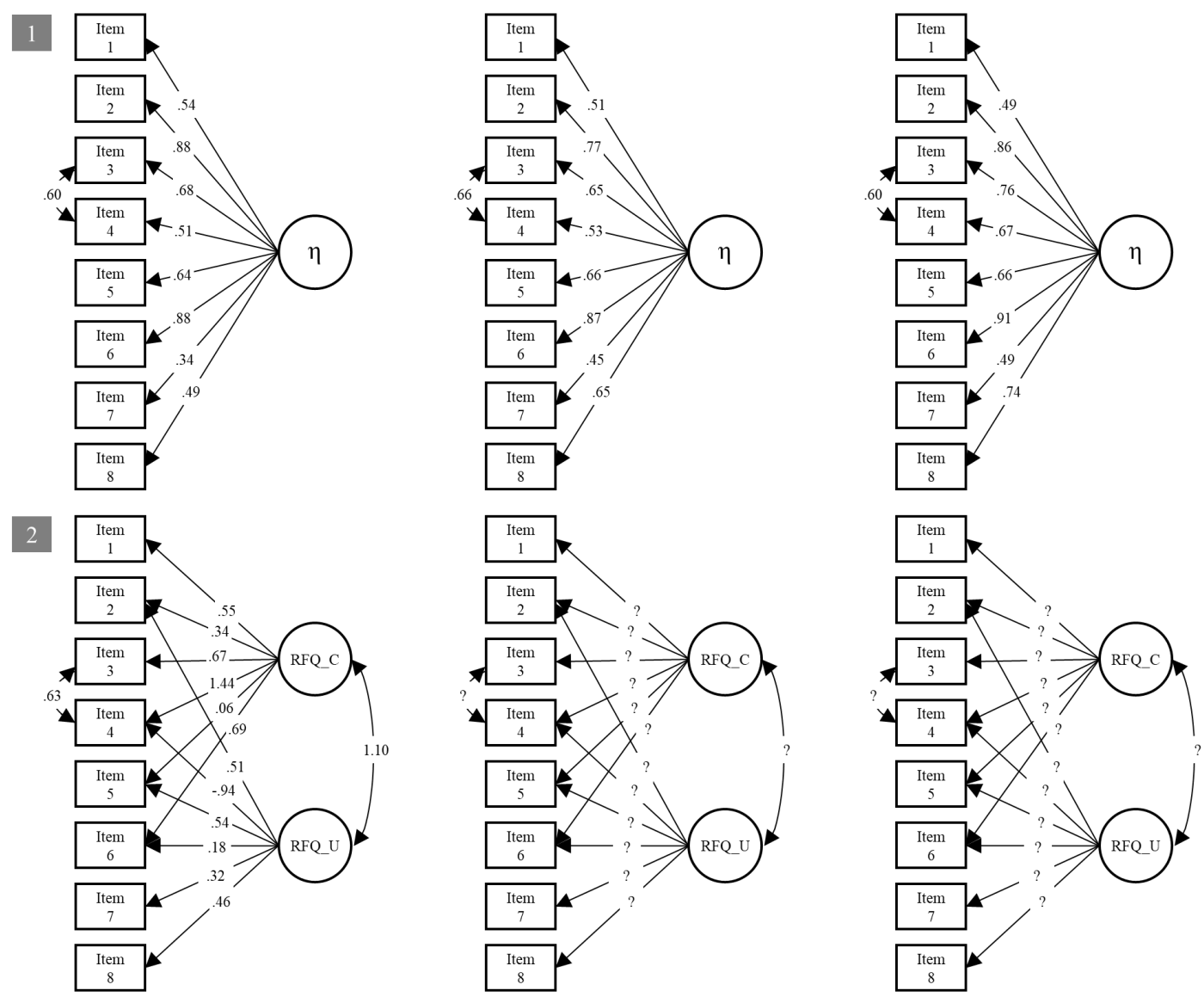

3
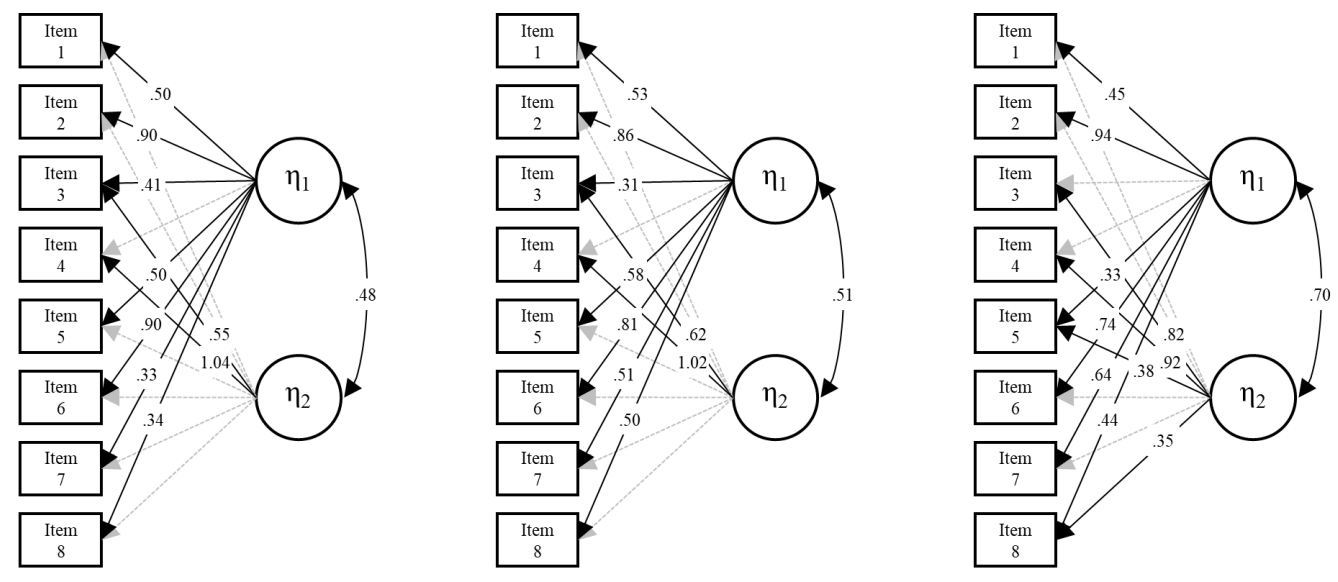

Note. All estimates are standardized. Loadings smaller than .30 are grayed out. In Sample 1, only the parameter estimates for admission are displayed. $1=$ Unidimensional CFA model, 2 = Two-dimensional CFA model, 3 = Two-dimensional EFA model; RFQ_C = Certainty About Mental States; RFQ_U = Uncertainty About Mental States; $\eta=$ RFQ factor. 


\section{Figure 3}

Bifactor Exploratory Structural Equation Model in Sample 1 at Admission (Panel A) and Sample 2 (Panel B)

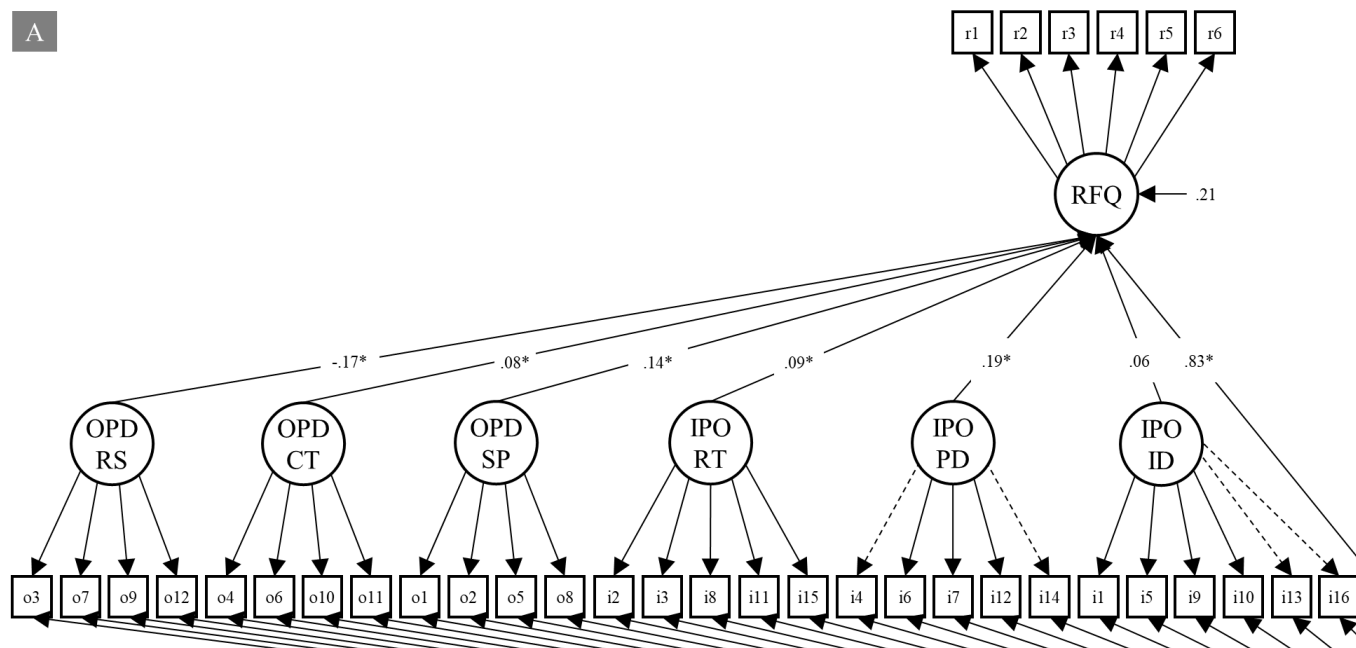

B

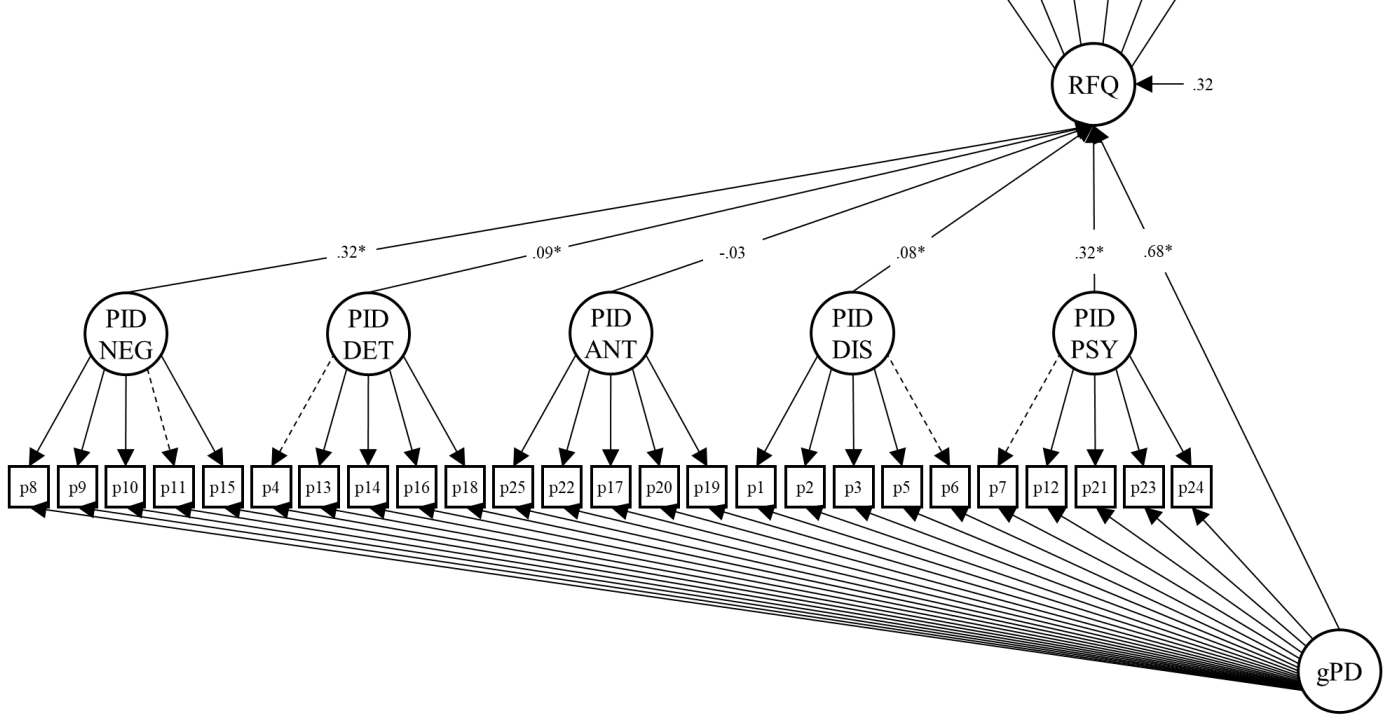

Note. All estimates are standardized. Intercepts, thresholds, and non-target loadings are not displayed. Non-target loadings were all $<.30$. Target loadings $<.30$ are indicated by dashed lines. o1-o12 = OPD-SQS items; i1-i16 = IPO-16 items; r1-r6 = RFQ-6 items; 1 1-p25 = PID-5-BF items; OPD RS = Relationship; OPD CT = Conflict; OPD SP = Self-Perception; IPO RT $=$ RealityTesting; IPO PD = Primitive Defenses; IPO ID = Identity Diffusion; NEG = Negative Affectivity; DET $=$ Detachment ANT $=$ Antagonism $;$ DIS $=$ Disinhibition $;$ PSY $=$ Psychoticism; $g P D=$ General Personality Pathology.

$* p<.05$ 


\section{Online Supplement}

\section{Note S1}

Further Information About Sample 1

In Sample 1, participants varied with regard to their educational background with $18 \%$ holding a university degree, $30 \%$ holding the higher education entrance qualification (i.e., the highest German school degree; “Abitur”), and 48\% reporting 10 years of schooling or less. Patients were diagnosed using clinical judgements based on the tenth edition of the International Classification of Diseases (ICD-10; World Health Organization [WHO], 2004). The most frequent group of diagnoses was that of depressive disorders (93\%), followed by anxiety disorders (42\%), personality disorders (39\%), substance use disorders (20\%), and somatoform disorders (18\%). Personality disorders (PD) included borderline PD (11\%), avoidant PD (11\%), dependent PD (2\%), and PD not otherwise specified (15\%). As is commonly found, patients exhibited a high level of comorbidity ( $82 \%$ more than one diagnosis, $54 \%$ more than two, $21 \%$ more than three, $5 \%$ more than four).

\section{Note S2}

Further Information About Sample 2

In Sample 2, participants had a rather high level of education with $41 \%$ of participants holding a university degree and an additional 37\% holding the "Abitur" certificate.

Participants indicated via self-report whether they were acutely suffering from a mental health condition. Twenty percent of the participants reported being affected by any mental disorder. Of these, more than half indicated an affective disorder (52\%), followed by borderline personality disorder (16\%) and post-traumatic stress disorder (9\%). As the university's website might also be a reference source for psychiatric inpatients of the university hospital that specializes in the treatment of severe trauma, it is possible that such inpatients were attracted to participate in the study via this route. 


\section{Note S3}

Bifactor ESEM Model Specification

In an exploratory bifactor measurement model (i.e., bifactor EFA), all indicators should load on a general factor and, at the same time, they should load on at least one of at least two multiple specific factors. In contrast to classic bifactor models (i.e., bifactor CFA; Holzinger \& Swineford, 1937), indicators are allowed to load on multiple specific factors. The bifactor ESEM used here involves a unidimensional confirmatory measurement model for the RFQ and an exploratory measurement model with orthogonal target rotation for indicators of personality dysfunction. In Sample 1, the indicators of IPO-16 and OPD-SQS were target rotated such that the loadings of indicators were targeted towards a general factor reflecting general personality dysfunction (GPD) and were also targeted towards their respective specific factors in alignment with the six assumed content domains or scales of the measures (i.e., IPO-16: identity diffusion, primitive defenses, and reality testing; Zimmermann et al., 2013; OPD-SQS: self-perception, contact, and relationship; Ehrenthal et al., 2015). In Sample 2, the indicators of PID-5-BF were target rotated in the same vein towards a general factor (gPD) and the five PID domains as specific factors (i.e., negative affectivity, antagonism, detachment, disinhibition, psychoticism; APA, 2013).

American Psychiatric Association (2013). Diagnostic and statistical manual of mental disorders (DSM-5®). American Psychiatric Pub.

Ehrenthal, J. C., Dinger, U., Schauenburg, H., Horsch, L., Dahlbender, R. W., \& Gierk, B. (2015). Entwicklung einer Zwölf-Item-Version des OPD-Strukturfragebogens (OPD-SFK) [Development of a 12-item version of the OPD-Structure Questionnaire (OPD-SQS)]. Zeitschrift für Psychosomatische Medizin und Psychotherapie, 61, 262-274. https://doi.org/10.13109/zptm.2015.61.3.262

Holzinger, K. J., \& Swineford, F. (1937). The bi-factor method. Psychometrika, 2, 41-54.

Zimmermann, J., Benecke, C., Hörz, S., Rentrop, M., Peham, D., Bock, A., Wallner, T., Schauenburg, H., Frommer, J., Huber, D., Clarkin, J. F., \& Dammann, G. (2013). Validierung einer deutschsprachigen 16Item-Version des Inventars der Persönlichkeitsorganisation (IPO-16) [Validity of a German 16-itemversion of the Inventory of Personality Organization (IPO-16)]. Diagnostica, 59, 3-16. https://doi.org/10.1026/0012-1924/a000076 


\section{Note S4}

Further Information About Sample 3

To identify careless respondents and thereby ensure data quality, we implemented a number of validity checks into the data collection process. Whereas attention checks are useful to detect careless responding in general, language fluency tests specifically target participants who participate by misrepresenting their language proficiency or place of residence. We employed two instructed response items that required participants to mark a specific response option that was clearly stated in the item instruction (e.g., "If you are paying attention, mark 'strongly agree'. Otherwise, you will be disqualified”). Thirty-four participants failed at least one of the two instructed response items and were excluded. In addition, we employed a language fluency check to ensure that participants met the participation requirement in terms of language proficiency (i.e., being fluent in English). We used an open-ended question at the end of the study that tasked participants with providing a statement on an opinion question. Four participants were excluded for failing to provide a proper statement.

In Sample 3, participants varied with regard to their educational level (e.g., 64\% with a bachelor's degree or higher) and occupational status (e.g., 57\% employed for wages). 


\section{Note S5}

Commonality Analysis Using the RFQ-8

Using the first set of variables, the association between the RFQ- 8 and LPFS-BF $\left(R^{2}=\right.$ $.48)$ was decomposed into variance shared with measures of mentalizing $\left(\Delta R^{2}=.25,95 \% \mathrm{CI}\right.$ $[.16 ; .30], p<.001)$, variance shared with measures of emotional lability and impulsivity alone $\left(\Delta R^{2}=.20,95 \% \mathrm{CI}[.15 ; .32], p<.001\right)$, and variance uniquely explained by LPFS-BF $\left(\Delta R^{2}=.03,95 \% \mathrm{CI}[.02 ; .05], p<.001\right)$. In the second set of variables, the association between the RFQ-8 and IPO-16 $\left(R^{2}=.42\right)$ was decomposed into variance shared with measures of mentalizing $\left(\Delta R^{2}=.32,95 \%\right.$ CI $\left.[.23 ; .37], p<.001\right)$, variance shared with measures of emotional lability and impulsivity alone $\left(\Delta R^{2}=.06,95 \%\right.$ CI $\left.[.04 ; .18], p<.001\right)$, and variance uniquely explained by IPO-16 $\left(\Delta R^{2}=.03,95 \% \mathrm{CI}[.01 ; .05], p<.001\right)$. On average, across all 32 combinations of predictors, the association between the RFQ- 8 and broad indicators of personality dysfunction (mean $R^{2}=.44$ ) was decomposed into variance shared with measures of mentalizing (mean $\Delta R^{2}=.28$ ), variance shared with measures of emotional lability and impulsivity alone (mean $\Delta R^{2}=.13$ ), and variance uniquely explained by IPO-16 (mean $\Delta R^{2}=.03$ ). Thus, $63 \%$ of the observed associations between RFQ-8 and indicators of personality dysfunction were due to variance shared with other measures of mentalizing, whereas $30 \%$ were due to variance shared with measures of emotional lability and impulsivity alone and $7 \%$ were unique to measures of personality dysfunction. 


\section{Table S1}

Bivariate Correlations Between $R F Q-8, R F Q \_C, R F Q \_U$, and Criterion Measures in Sample 1 at Admission

\begin{tabular}{|c|c|c|c|c|c|c|c|c|c|}
\hline & RFQ-8 & RFQ_C & RFQ_U & BSI & PHQ-15 & PHQ-9 & IIP-32 & IPO-16 & OPD-SFK \\
\hline RFQ_C & -.92 & & & & & & & & \\
\hline RFQ_U & .80 & -.65 & & & & & & & \\
\hline BSI & .55 & -.46 & .53 & & & & & & \\
\hline PHQ-15 & .26 & -.23 & .25 & .59 & & & & & \\
\hline PHQ-9 & .42 & -.34 & .43 & .76 & .47 & & & & \\
\hline IIP-32 & .54 & -.48 & .48 & .63 & .32 & .51 & & & \\
\hline IPO-16 & .72 & -.68 & .62 & .59 & .33 & .46 & .60 & & \\
\hline OPD-SFK & .65 & -.57 & .60 & .72 & .41 & .59 & .68 & .69 & \\
\hline WHO-5 & -.23 & .18 & -.24 & -.51 & -.29 & -.65 & -.35 & -.22 & -.38 \\
\hline
\end{tabular}

Note. $N=861$. All $p<.001$. 
Table S2

Bivariate Correlations Between $R F Q-8, R F Q \_C, R F Q_{-} U$, and Criterion Measures in Sample 2

\begin{tabular}{|c|c|c|c|c|c|c|c|c|}
\hline & RFQ-8 & RFQ_C & RFQ_U & Total & NEG & DET & ANT & DIS \\
\hline RFQ_C & -.91 & & & & & & & \\
\hline RFQ_U & .88 & -.64 & & & & & & \\
\hline PID-5-BF Total Score & .67 & -.61 & .60 & & & & & \\
\hline Negative Affectivity (NEG) & .60 & -.52 & .56 & .73 & & & & \\
\hline Detachment (DET) & .46 & -.43 & .39 & .72 & .43 & & & \\
\hline Antagonism (ANT) & .30 & -.32 & .23 & .65 & .29 & .29 & & \\
\hline Disinhibition (DIS) & .44 & -.40 & .41 & .73 & .40 & .36 & .43 & \\
\hline Psychoticism (PSY) & .62 & -.56 & .59 & .82 & .52 & .52 & .44 & .50 \\
\hline
\end{tabular}

Note. $N=566$. All $p<.001$. 


\section{Table S3}

Bivariate Correlations Between $R F Q-8, R F Q \_C, R F Q \_U$, and Further Measures in Sample 3

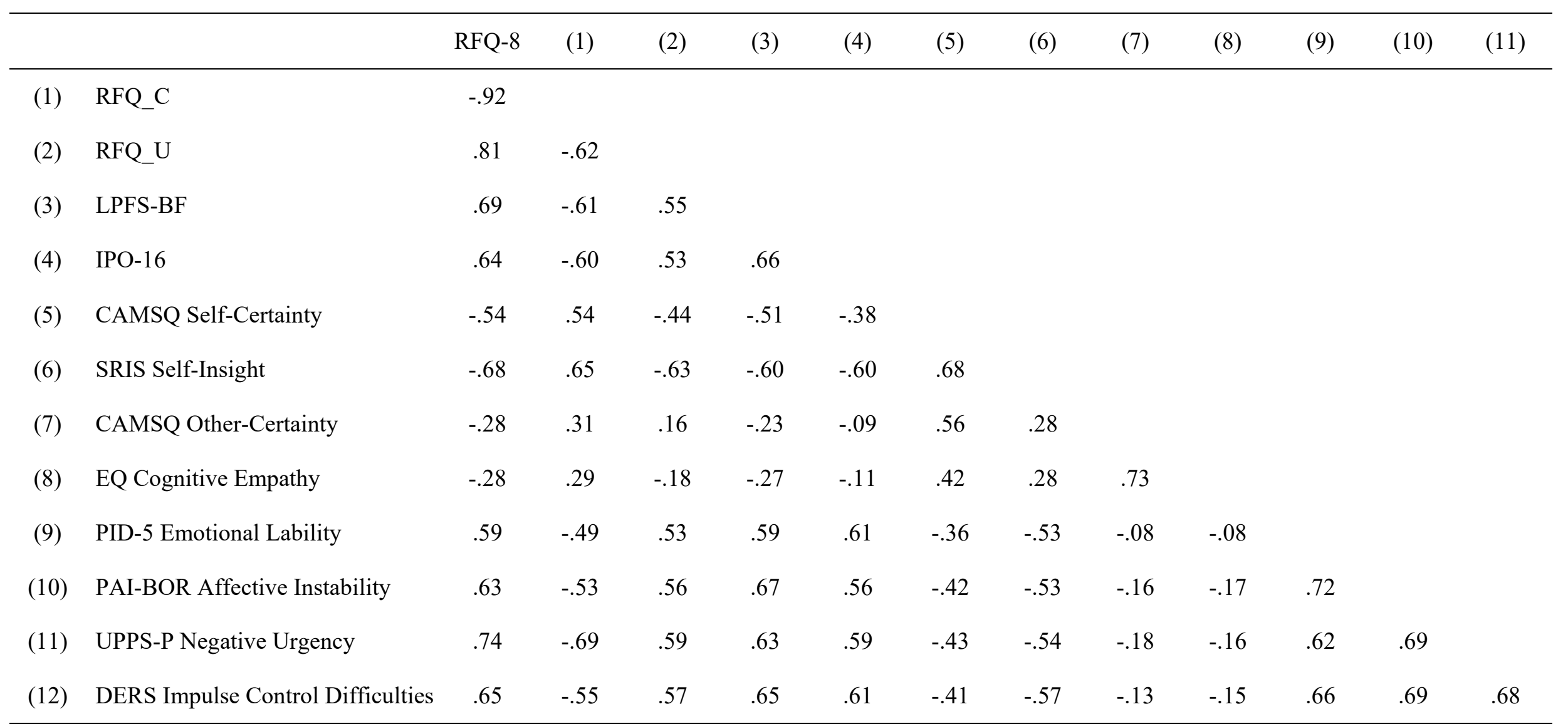

Note. $N=862$. All correlations are statistically significant at $p<.05$. 


\section{Figure S1}

Originally Proposed Two-Dimensional CFA Model Using Double-Scoring in Sample 1 at Admission

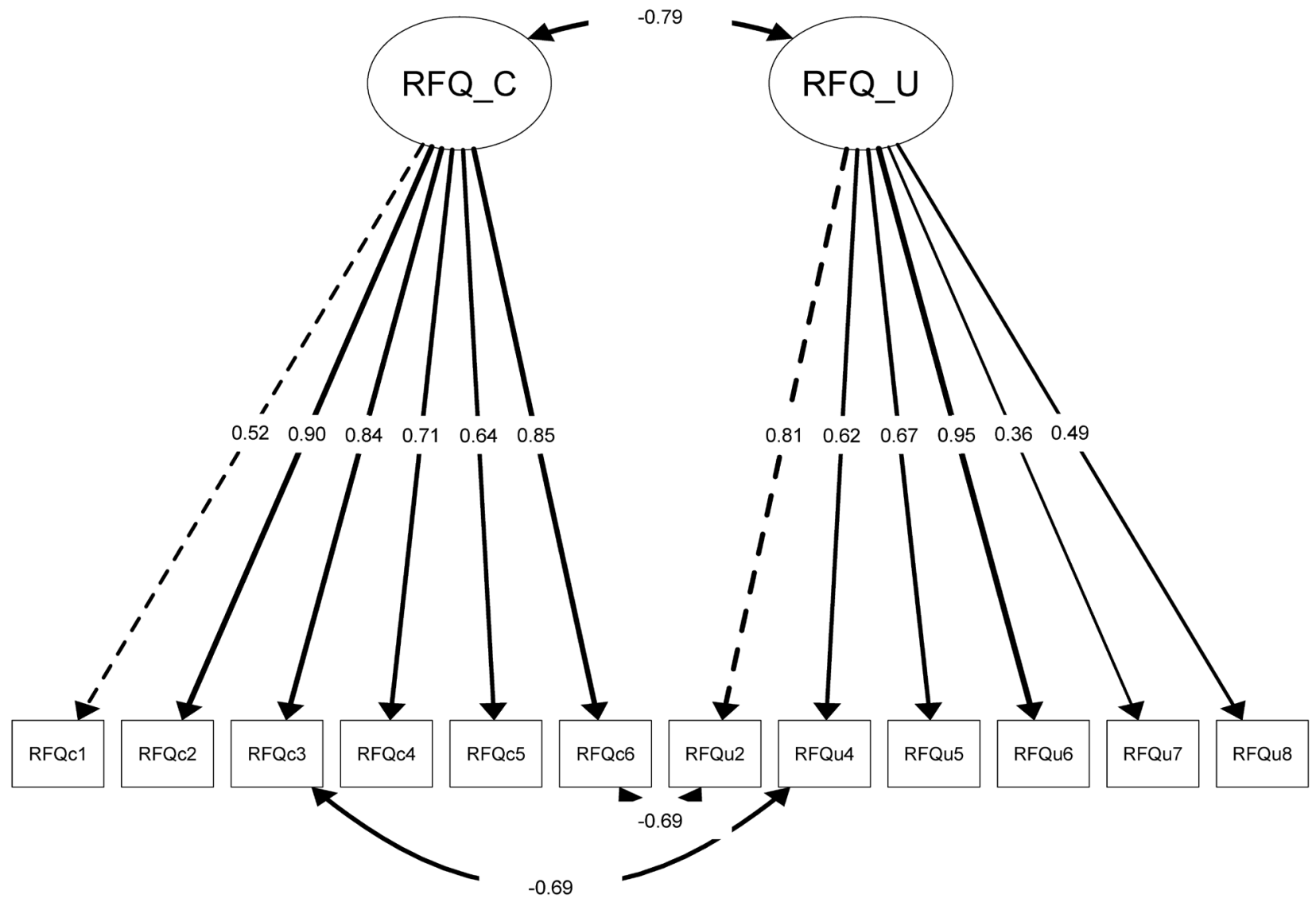

Note. $N=861 . \chi^{2}(51)=490.05, \mathrm{CFI}=.95, \mathrm{RMSEA}=.10, \mathrm{SRMR}=.15 . \omega=.88 / .73(\mathrm{RFQ}$ C $/ \mathrm{RFQ}$ U $)$.

The model was estimated using the WLSMV estimator. Correlated errors were specified following

recommendations by Fonagy et al. (2016). Intercepts, residual variances, and thresholds are not displayed. 


\section{Figure S2}

Originally Proposed Two-Dimensional CFA Model Using Double-Scoring in Sample 2

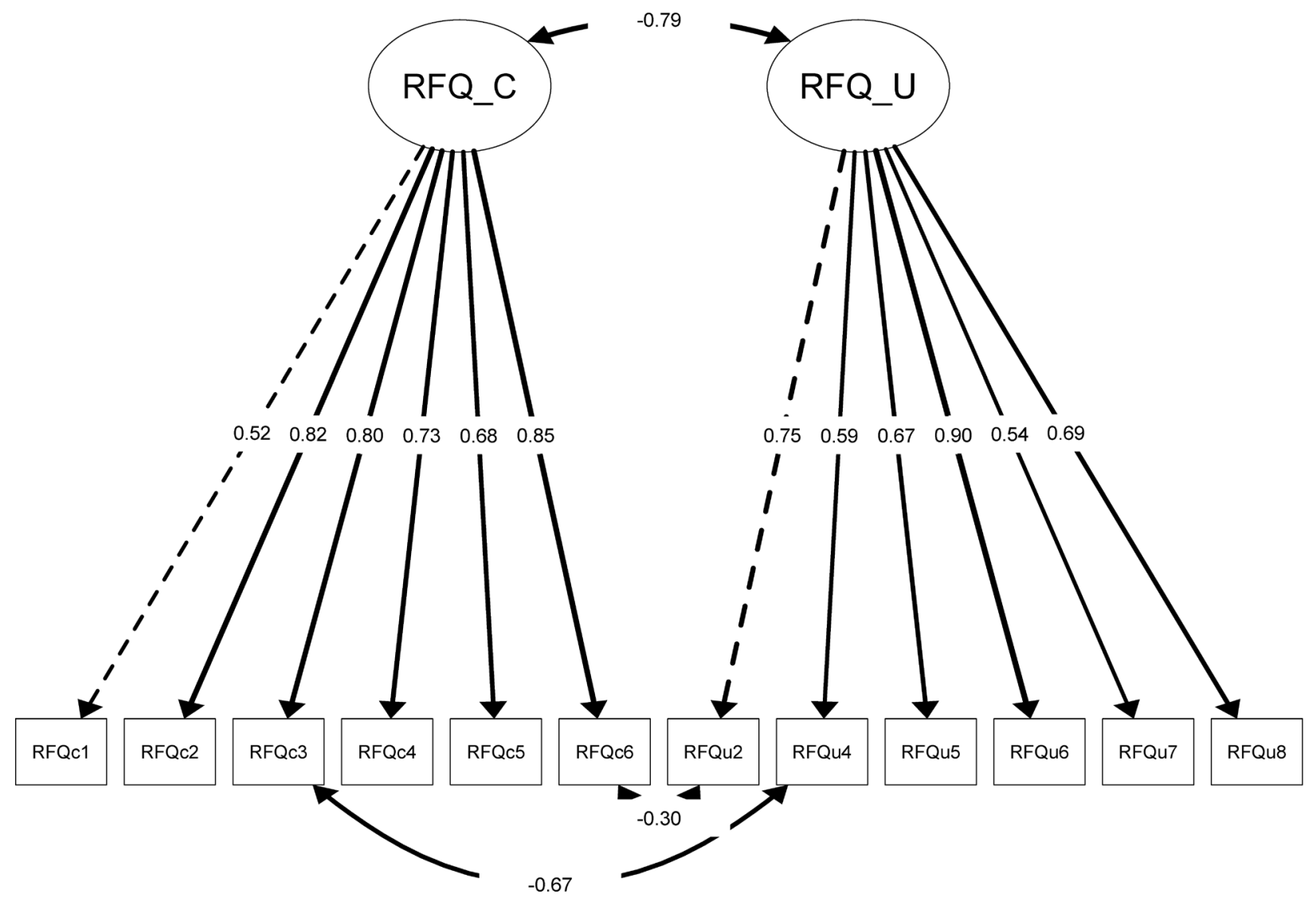

Note. $N=566 . \chi^{2}(51)=313.46, \mathrm{CFI}=.95, \mathrm{RMSEA}=.10, \mathrm{SRMR}=.15 . \omega=.88 / .78(\mathrm{RFQ} \mathrm{C} / \mathrm{RFQ} \mathrm{U})$. The model was estimated using the WLSMV estimator. Correlated errors were specified following recommendations by Fonagy et al. (2016). Intercepts, residual variances, and thresholds are not displayed. 
Figure S3

Lowess-Smoothed Regression Curves of the Association Between the RFQ-6 and Criterion Measures in Sample 1 at Admission
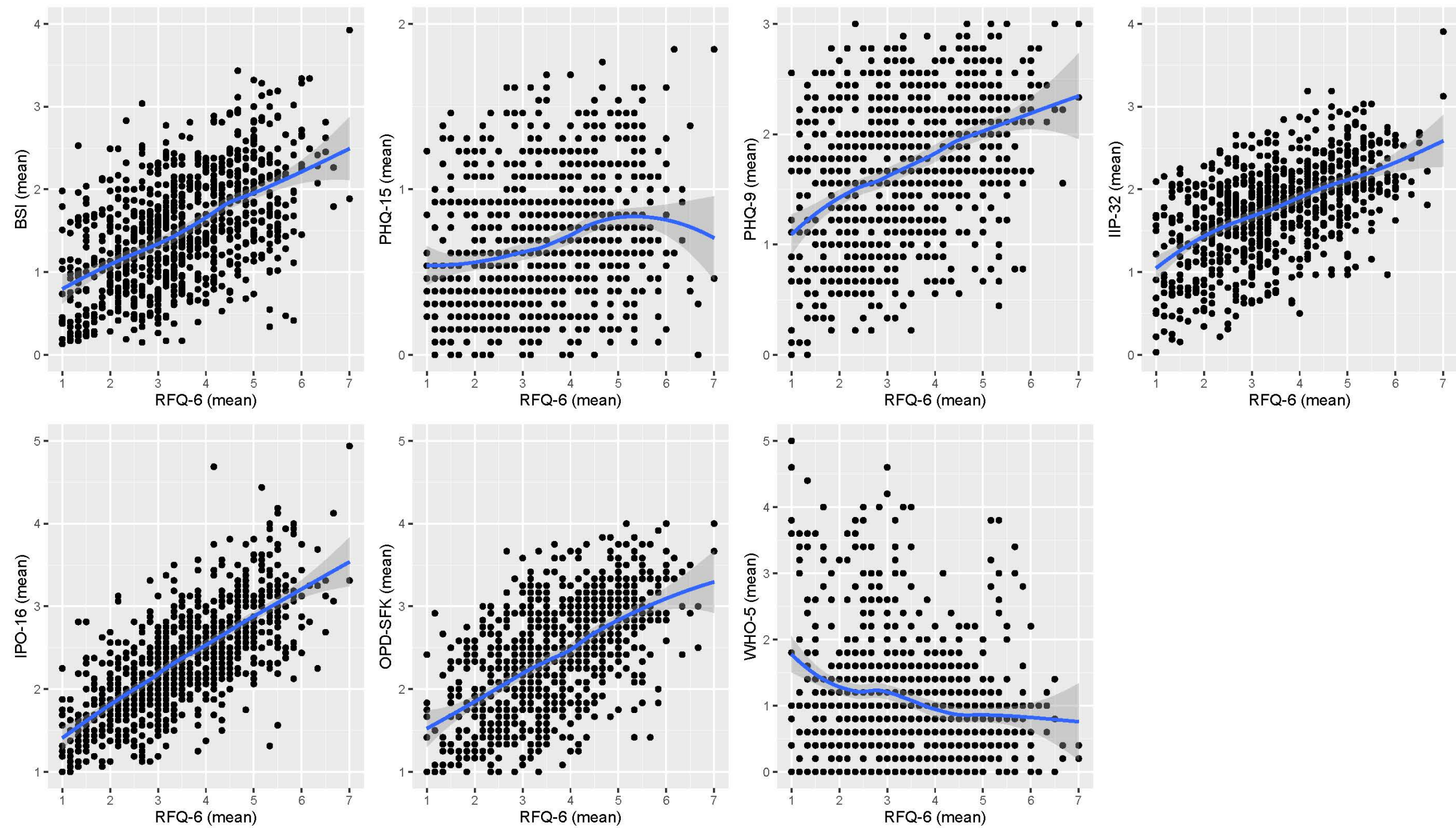
Figure S4

Lowess-Smoothed Regression Curves of the Association Between the RFQ-6 and Criterion Measures in Sample 2
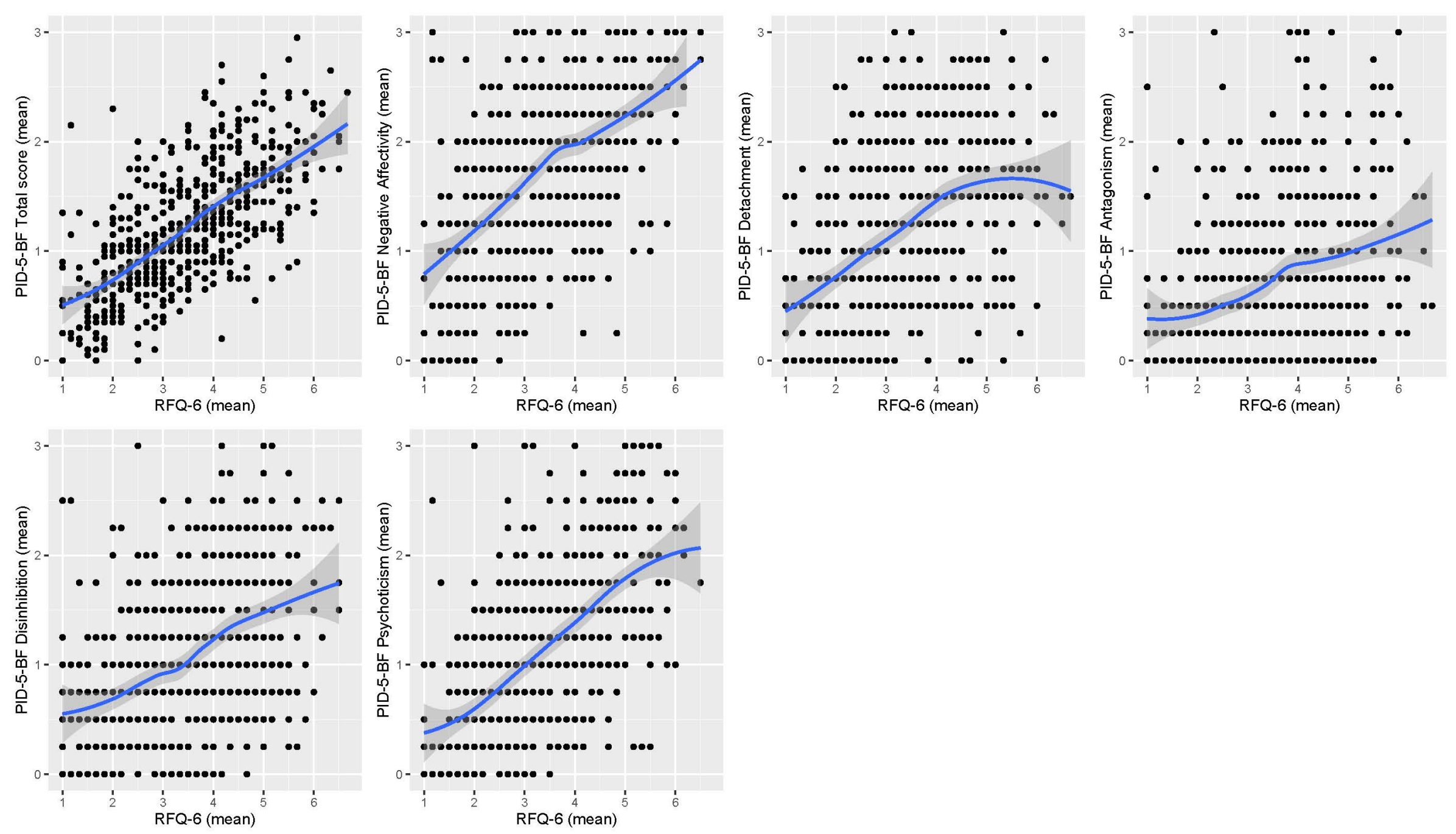


\section{Figure S5}

Two-Lines Test of the Association Between the RFQ-6 and Criterion Measures in Sample 1 at Admission
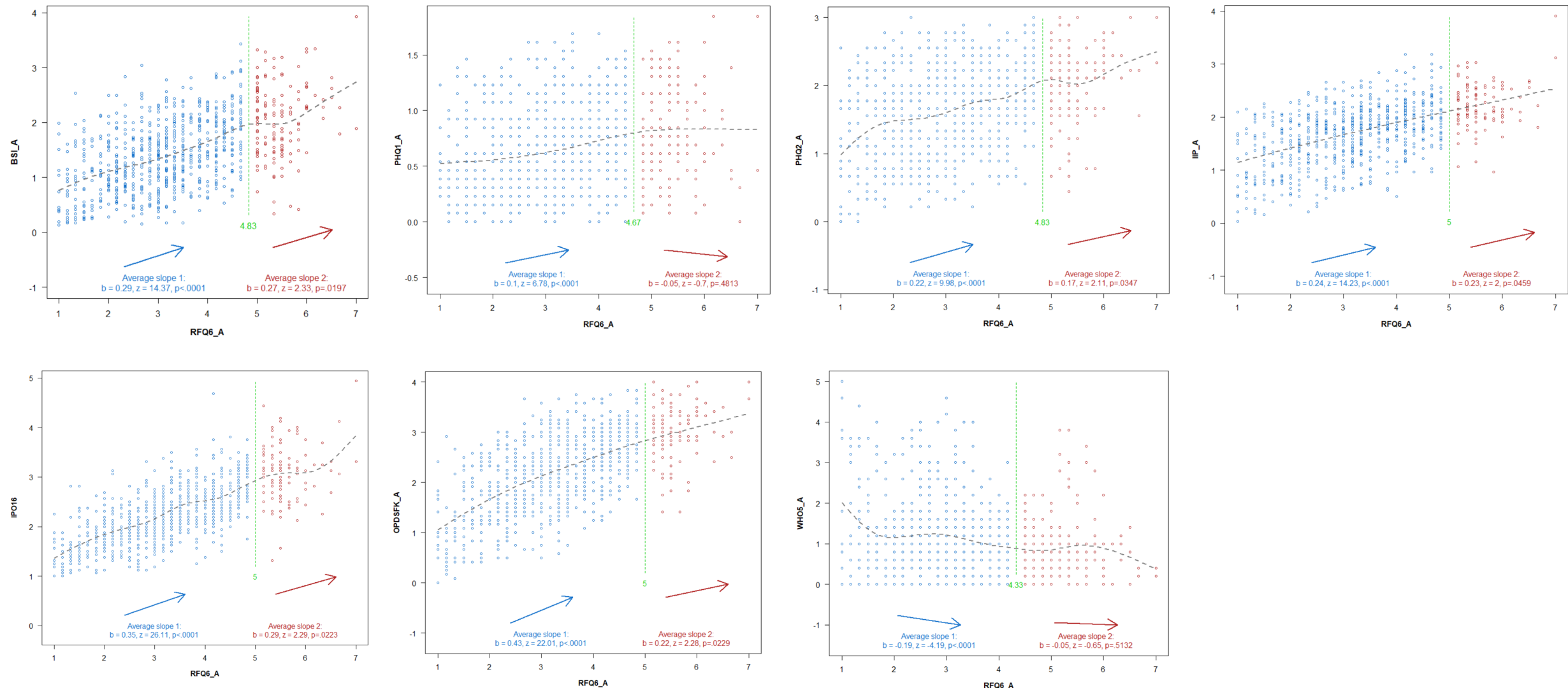


\section{Figure S6}

Two-Lines Test of the Association Between the RFQ-6 and Criterion Measures in Sample 2
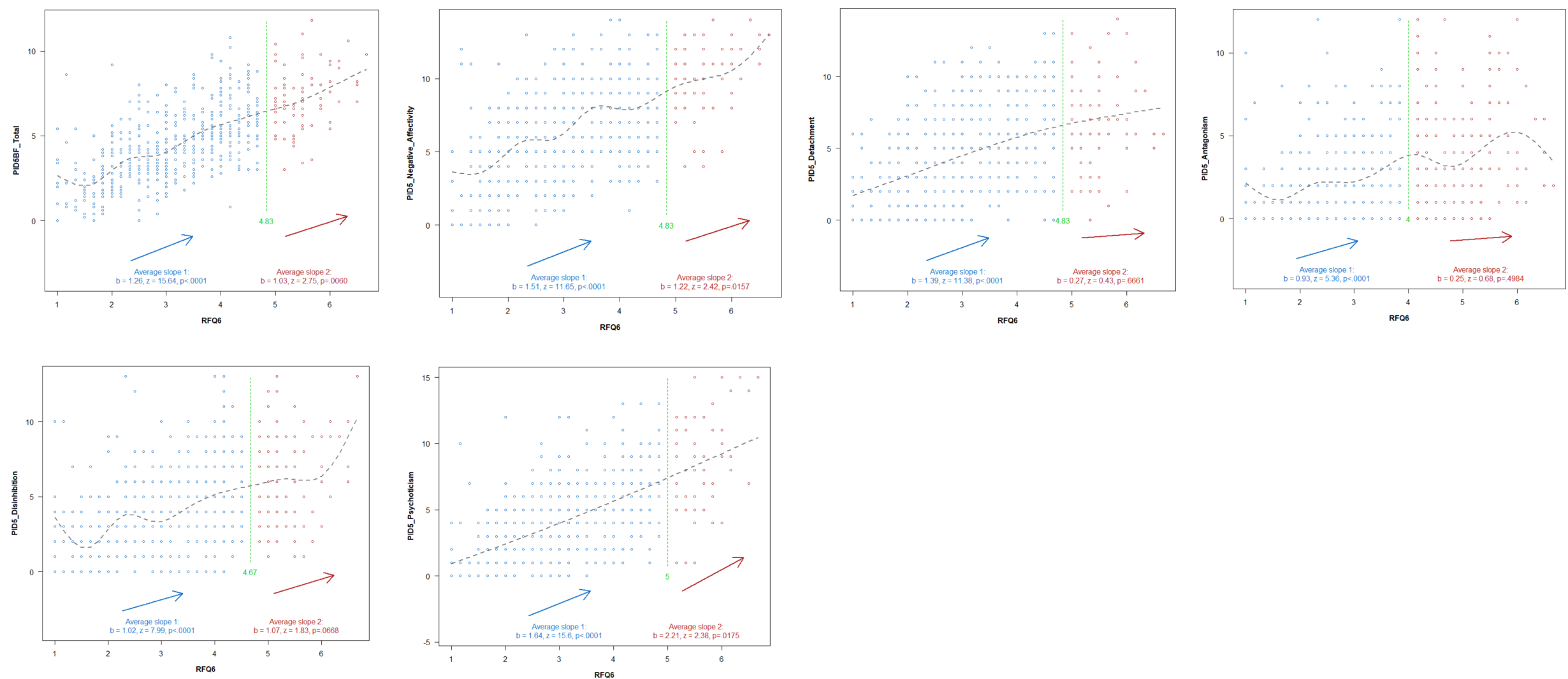


\section{Figure S7}

Bifactor Exploratory Structural Equation Model in Sample 1 at Admission (Panel A) and

Sample 2 (Panel B) Using all Eight Items of the RFQ

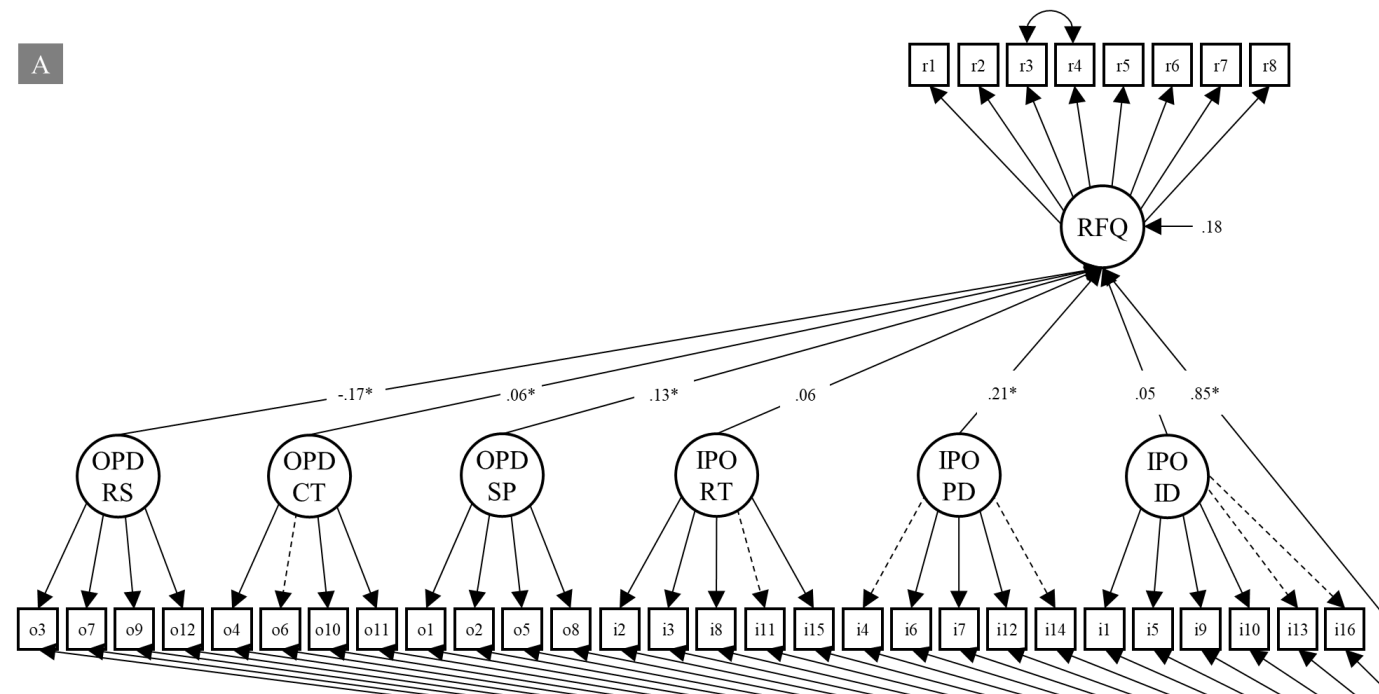

B
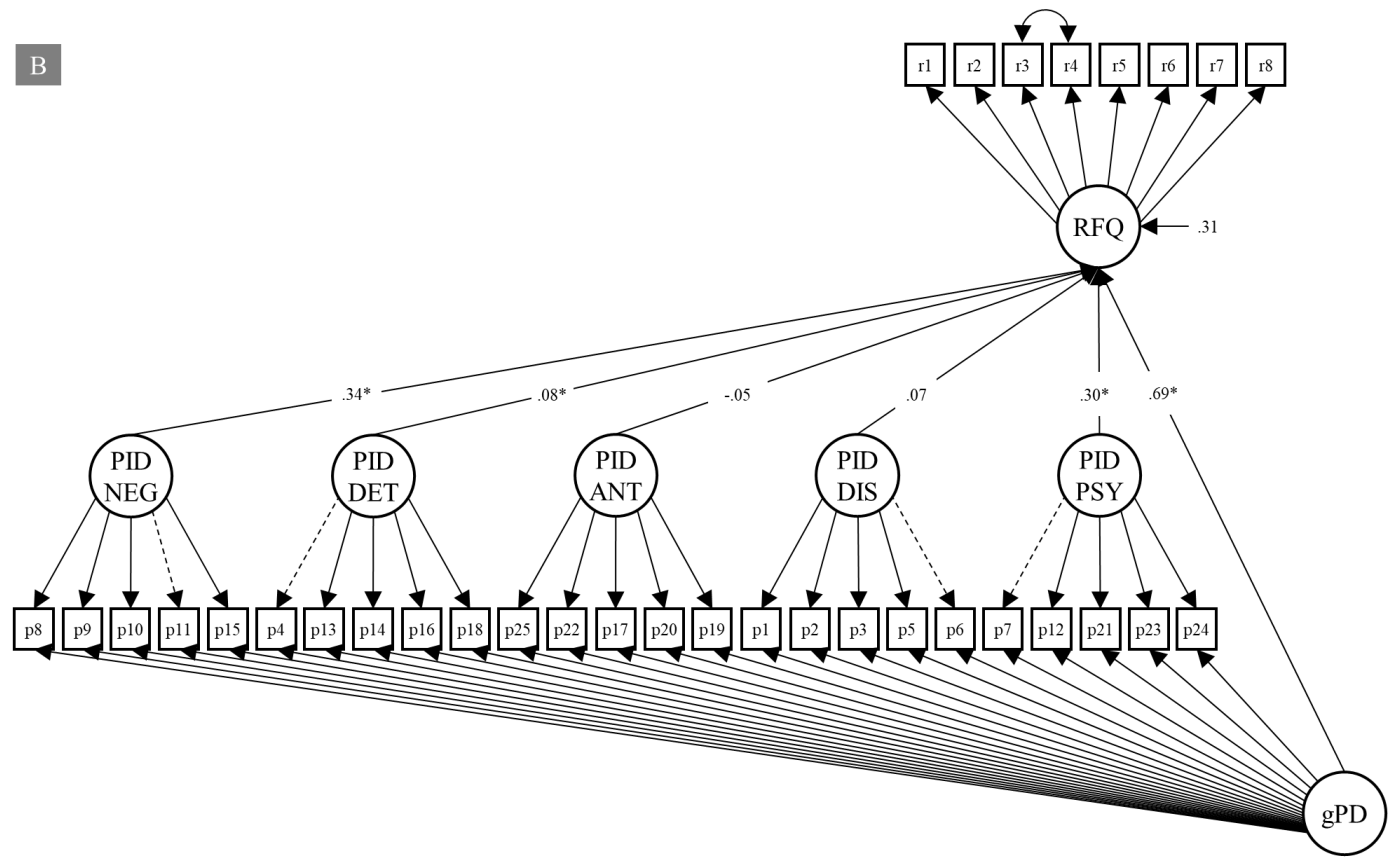

Note. All estimates are standardized. Intercepts, thresholds, and non-target loadings are not displayed. Non-target loadings were all $<.30$. Target loadings $<.30$ are indicated by dashed lines. Variable names are the same as in Figure 3. In Sample 1, model fit was CFI $=.94$, RMSEA $=.06$, $\mathrm{SRMR}=.04$. In Sample 2, model fit was CFI $=.96, \mathrm{RMSEA}=.05, \mathrm{SRMR}=.04$.

$* p<.05$ 